\title{
Mesoscale Processes during the Genesis of Hurricane Karl (2010)
}

\author{
MiCHAEL M. BELL \\ Colorado State University, Fort Collins, Colorado \\ MiCHAEL T. MONTGOMERY \\ Department of Meteorology, Naval Postgraduate School, Monterey, California
}

(Manuscript received 6 June 2018, in final form 9 March 2019)

\begin{abstract}
Observations from the Pre-Depression Investigation of Cloud Systems in the Tropics (PREDICT), Genesis and Rapid Intensification Processes (GRIP), and Intensity Forecast Experiment (IFEX) field campaigns are analyzed to investigate the mesoscale processes leading to the tropical cyclogenesis of Hurricane Karl (2010). Research aircraft missions provided Doppler radar, in situ flight level, and dropsonde data documenting the structural changes of the predepression disturbance. Following the pre-Karl wave pouch, variational analyses at the meso- $\beta$ and meso- $\alpha$ scales suggest that the convective cycle in Karl alternately built the low- and midlevel circulations leading to genesis episodically rather than through a sustained lowering of the convective mass flux from increased stabilization. Convective bursts that erupt in the vorticity-rich environment of the recirculating pouch region enhance the low-level meso- $\beta$ - and meso- $\alpha$-scale circulation through vortex stretching. As the convection wanes, the resulting stratiform precipitation strengthens the midlevel circulation through convergence associated with ice microphysical processes, protecting the disturbance from the intrusion of dry environmental air. Once the column saturation fraction returns to a critical value, a subsequent convective burst below the midlevel circulation further enhances the low-level circulation, and the convective cycle repeats. The analyses suggest that the onset of deep convection and associated low-level spinup were closely related to the coupling of the vorticity and moisture fields at low and midlevels. Our interpretation of the observational analysis presented in this study reaffirms a primary role of deep convection in the genesis process and provides a hypothesis for the supporting role of stratiform precipitation and the midlevel vortex.
\end{abstract}

\section{Introduction}

An understanding of tropical storm formation requires insight into processes occurring at multiple spatial and temporal scales. A synoptic-scale tropical wave can provide a protective environment and background vorticity for a predepression, but does not guarantee that the predepression will intensify into a strong tropical cyclone (Frank 1970; Dunkerton et al. 2009, hereafter DMW09). Processes on the meso- $\gamma(\sim 2-20 \mathrm{~km})$, meso- $\beta$ $(\sim 20-200 \mathrm{~km})$, and meso- $\alpha(\sim 200-1000 \mathrm{~km})$ scales within the predepression must concentrate enough planetary vorticity in the lower troposphere to organize convection and sufficiently increase the rotational wind speeds to be declared a tropical depression or tropical storm. The processes by which this occurs are the primary focus of this paper.

Corresponding author: Michael M. Bell, mmbell@colostate.edu
While spontaneous tropical cyclone (TC) formation can occur in idealized numerical simulations (e.g., Nolan et al. 2007), cyclogenesis in the real atmosphere occurs within a precursor synoptic-scale disturbance such as a tropical wave with favorable large-scale atmospheric and oceanic conditions (Gray 1968; McBride and Zehr 1981). A new model for cyclogenesis in tropical waves developed by DMW09 describes a recirculation region within the critical layer of the parent wave where air tends to be repeatedly moistened by cumulus convection, protected to some degree from lateral intrusion of dry air and deformation by horizontal or vertical shear, and (thanks to its location near the critical level) able to keep pace with the parent wave until the dominant

Publisher's Note: This article was revised on 20 November 2019 to correct a typographical error that caused extra text to be included in the abstract when originally published. 
vortex has strengthened into a self-maintaining entity. The combination of the associated genesis sequence and the overarching framework for describing how such hybrid wave-vortex structures become tropical depressions is likened to the development of a marsupial infant in its mother's pouch (see DMW09; Montgomery et al. 2010; Wang et al. 2010a,b).

In late summer 2010 the Pre-Depression Investigation of Cloud Systems in the Tropics (PREDICT) field campaign was conducted to improve our understanding of how tropical waves transition into tropical cyclones and test the principal hypothesis that tropical storm formation is greatly favored in the critical-layer region of the synoptic-scale predepression disturbance (Montgomery et al. 2012). The National Science Foundation (NSF) supported the PREDICT field campaign, which involved 25 research flights into Atlantic tropical disturbances. The PREDICT campaign was conducted in conjunction with NASA Genesis and Rapid Intensification Processes (GRIP) and National Oceanic and Atmospheric Administration (NOAA) Intensity Forecast Experiment (IFEX) campaigns.

Composite analyses of developing and nondeveloping disturbances ("developers" and "nondevelopers") using the PREDICT dataset have revealed distinctions between favorable and unfavorable thermodynamic environments for genesis. Montgomery and Smith (2012), Davis and Ahijevych (2013), and Komaromi (2013) showed that development depends strongly on available moisture above the boundary layer. Developers had higher equivalent potential temperature $\theta_{e}$ in the low to midtroposphere due to both warmer and moister air, while nondevelopers had high convective available potential energy (CAPE) relative to the developers due to cooler and drier air at midlevels. In particular, Smith and Montgomery (2012) found that "the most prominent difference between the non-developing system [ex-Gaston] and the two developing systems [preKarl and pre-Matthew] was the much larger reduction of $\theta_{e}$ between the surface and a height of $3 \mathrm{~km}$, typically $25 \mathrm{~K}$ in the non-developing system, compared with only $17 \mathrm{~K}$ in the developing systems. Conventional wisdom would suggest that, for this reason, the convective downdrafts would be stronger in the non-developing system and would thereby act to suppress the development."

Smith and Montgomery (2012) proposed an alternative hypothesis "in which the drier air weakens the convective updrafts and thereby the convective amplification of absolute vorticity necessary for development." This alternative hypothesis was tested by Freismuth et al. (2016) for the nondeveloping case of ex-Gaston using largescale analyses. Freismuth et al. showed that "entrained, dry air near $600 \mathrm{hPa}$ thwarted convective updrafts and vertical mass flux, which in turn led to a reduction in vorticity and a compromised pouch at these middle levels. A compromised pouch allows further intrusion of dry air and quenching of subsequent convection, therefore hindering vorticity amplification through vortex tube stretching." Gjorgjievska and Raymond (2014) argued that a reduction in midlevel vorticity may have occurred prior to the ingestion of dry air, which initially weakened the pouch and allowed for subsequent intrusion of dry air. The findings of both Freismuth et al. (2016) and Gjorgjievska and Raymond (2014) point to a potentially important positive role of the midlevel cyclonic circulation by providing protection against dry-air intrusions from the complex environment and supporting a favorable region for persistent convective activity and vortex tube stretching on the system-scale circulation.

Numerical simulations and large-scale analyses by Montgomery et al. (2010), Wang et al. (2010a, 2012), Wang (2012), and others have now provided abundant evidence that a deep, moist pouch is a necessary condition for the development of a tropical disturbance in the Atlantic basin, but the specific role of the midlevel and low-level circulations, enhanced temperature and moisture at midlevels, and the relative importance of deep, congestus, and stratiform convection in the development process are still under debate. As an example of the diversity of thought on the key ingredients of the genesis process, a competing theory on the role of the midlevel circulation has been proposed by D. Raymond and coworkers. The idea is that the enhanced static stability associated with a midlevel cyclonic vortex is an essential element of developers (Raymond and Sessions 2007; Raymond et al. 2011; Raymond 2012; Davis and Ahijevych 2013; Zawislak and Zipser 2014; Gjorgjievska and Raymond 2014). This theory emphasizes the thermodynamic structure of the tropical disturbance, revising an early emphasis on stratiform mesoscale convective systems (MCSs) that emerged from prior field campaigns two decades earlier (e.g., Bister and Emanuel 1997; Raymond et al. 1998; Simpson et al. 1997). The work of Raymond et al. (2011) and Gjorgjievska and Raymond (2014) has proposed a refinement of the marsupial paradigm, suggesting that the enhanced stability causes a lowering of the convective mass flux and, by mass continuity, a spinup of the lower troposphere by the convergence of cyclonic vertical vorticity from the near environment. In the proposed refinement, the thermodynamic structure that is in balance with the vorticity provides a strong control on the humidity profile and convective mass flux through a "moisture quasi equilibrium" process (Raymond and Flores 2017).

Organized deep convection has been acknowledged as a necessary component of genesis for several decades 
(e.g., Gray 1968; Houze et al. 2009), and is in fact part of the definition of a TC according to the National Hurricane Center (see http://www.nhc.noaa.gov/aboutgloss.shtml). Observations suggest repeated bursts of vortical deep convection are an important component of the genesis process (Zehr 1992; Gjorgjievska and Raymond 2014; Raymond et al.1998; Chang et al. 2017). A key cloud-scale process identified by numerical studies (Hendricks et al. 2004; Montgomery et al. 2006; Nguyen et al. 2008; Zhang et al. 2011) is that low-level convergence driven by deep convection in a rotating environment amplifies preexisting vertical vorticity locally by vortex tube stretching, and that the amplified vorticity outlives the deep convection that produced it. Wissmeier and Smith (2011) and Kilroy and Smith (2013) showed that vertical vorticity could be amplified by an order of magnitude by even moderate, short-lived convective updrafts in idealized numerical simulations of tropical convection. Vortical remnants concentrated by deep convection will tend to aggregate within the broader cyclonic circulation, leading to a corresponding upscale vorticity and energy cascade and further amplification of vertical vorticity by subsequent convective bursts. The system-scale inflow forced by the aggregate latent heating from the convective elements leads to an inward advection of convectively enhanced vorticity. Stokes's theorem applied to a fixed area surrounding the convection implies that there will be an accompanying increase in the strength of the disturbance-scale circulation on account of the import of ambient absolute vorticity.

Observations have shown evidence for the presence of vortical deep convection in several TC genesis cases. Zipser and Gautier (1978) concluded that a giant updraft within an MCS accounted for the majority of upward mass flux in a tropical depression off the coast of Africa in 1974, and that the low-level convergence supporting the MCS could stretch the environmental vorticity at a rate that would account for much of the intensification of the depression. Further evidence showing the existence and operation of vortical deep convection has been found since those early observations (Reasor et al. 2005; Sippel et al. 2006; Houze et al. 2009; Bell and Montgomery 2010; Sanger et al. 2014). However, most of these observations consist of snapshots or short time samples, and it has been difficult using observations only to piece together the cumulative effects of deep convection in the pregenesis period. The comprehensive dataset obtained in predepression Karl (2010) provides a unique series of aircraft observations to help investigate the roles of convective and stratiform precipitation processes in TC genesis.

Observations from predepression Karl collected during PREDICT have been used in this study to quantify the dynamic and thermodynamic effects of deep convection, stratiform precipitation, and vortex intensification following the recirculating pouch region for 5 days leading up to genesis. Doppler radar, in situ, and dropsonde observations have been analyzed using a spline-based variational technique called Spline Analysis at Mesoscale Utilizing Radar and Aircraft Instrumentation (SAMURAI) (Bell et al. 2012) to examine the mesoscale structure. Similar to the results reported by Lussier et al. (2014), circulation tendencies suggest progressive development of the low-level circulation through low-level convergence of vorticity in deep convective bursts. A strong midlevel circulation develops also over this period, consistent with the hypothesis that stratiform precipitation processes also play an important role in genesis. Our results will suggest that divergence profiles associated with convectively inactive MCS regions in predepression Karl contributed to a low-level spindown of the vortex.

We propose here the hypothesis that the primary role of stratiform MCS regions is to promote midlevel convergence that enhances the midlevel meso- $\alpha$-scale pouch circulation. As anticipated by the foregoing discussions, the enhanced midlevel pouch region serves to recirculate moisture above the boundary layer in a frame of reference moving with the synoptic-scale disturbance. The enhanced moisture leads to decreased entrainment for subsequent bursts of deep convection (Holloway and Neelin 2009; James and Markowski 2010; Kilroy and Smith 2013) that can spin up the low-level circulation. This hypothesis is distinct from the emphasis in previous studies on the importance of the enhanced static stability leading to genesis. Our results suggest that the low-level cooling seen in previous composite analyses is episodic rather than persistent, and that the upper-level warming is associated with the developing warm-cored vortex.

In addition to the dynamic effect of enhancing the midlevel pouch, the direct effect of low-level moistening and cooling by stratiform rain from MCS regions is also beneficial, and may help the recovery of the low-level relative humidity after bursts of deep convection. A positive phasing between the low-level cooling and the diurnal cycle appears to have also contributed to Karl's genesis, consistent with the numerical study of Melhauser and Zhang (2014). Our analysis suggests that a critical value of saturation fraction for the layer below $700 \mathrm{hPa}$ near $85 \%$ relative humidity $(\mathrm{RH})$ allows for the onset of deep convection, similar to that analyzed in large-scale tropical convection (Neelin et al. 2009). The episodic nature of the convective bursts is tied closely to moisture availability above the boundary layer, consistent with the hypothesis of Holloway and Neelin (2009) that the deep convective onset is due to enhanced buoyancy of an entraining air parcel. The saturation fraction below $700 \mathrm{hPa}$ stays close to $85 \%$ in the days leading up to genesis, with repeated bursts of 
deep convection occurring when the meso- $\alpha$-scale environment exceeds this critical value, resulting in strong low-level convergence and spinup of the TC.

An outline of the remaining paper is as follows: Section 2 describes the PREDICT dataset and the analysis methodology used in this study. Results from the mesoscale analysis of Karl's genesis are presented in section 3. The circulation dynamics of the developing tropical cyclone are presented in section 4 . Section 5 summarizes the results and discusses their implications in the context of recent studies.

\section{Data and methodology}

The NSF-National Center for Atmospheric Research (NCAR) Gulfstream-V (GV) aircraft collected highaltitude $(\sim 13-14 \mathrm{~km})$ in situ and full-tropospheric dropsonde observations in a meso- $\alpha$-scale region encompassing the pouch and its nearby environment. $\mathrm{GV}$ in situ and dropsonde data were processed and quality controlled by the NCAR Earth Observing Laboratory. A correction for a dry bias in the relative humidity for dropsondes from 2010 was applied. Two NOAA WP-3Ds (P3s) were available for three missions that collected low-altitude $(\sim 3 \mathrm{~km})$ in situ and dropsonde observations, and added Doppler radar observations. The X-band $(3 \mathrm{~cm})$ wavelength tail Doppler radar (TDR) aboard the P3 aircraft employed a fore-aft scanning technique that provided radial velocity data in a cone $\sim 20^{\circ}$ from the track both fore and aft of the aircraft. Three-dimensional winds and precipitation structure were derived from the multiple Doppler velocities. Following the methodology developed by Bosart et al. (2002), the data were first corrected for navigational errors and manually edited to remove ocean returns, radar sidelobes, and other artifacts. The Doppler radar data were the only available tools to probe the three-dimensional convective structure of the pouch on the meso- $\gamma$ and meso- $\beta$ scales (Marks and Houze 1984; Reasor et al. 2005), but were only available around 0000 and 1200 UTC 13 and 0000 UTC 14 September. Limited radar echoes during the 0000 UTC 14 September P-3 mission precluded quantitative analysis of the convective structure at that time; therefore, the radar analysis presented herein focuses on 13 September.

The SAMURAI variational analysis package (Bell et al. 2012; Foerster et al. 2014; Lussier et al. 2014; Foerster and Bell 2017) was used to composite the research observations at horizontal node spacings of 25 and $2 \mathrm{~km}$ to examine the predepression structure at meso- $\alpha$ and meso- $\beta$ scales, respectively. One of the advantages of variational analyses such as SAMURAI is the capability to combine observations from different instruments with a prior estimate of the atmospheric state. For this study, the $0.25^{\circ}$ European Centre for Medium-Range Weather Forecasts (ECMWF) analyses were used as an initial estimate for the $25-\mathrm{km}$ Cartesian grid analyses. The SAMURAI meso- $\alpha$-scale analyses were then calculated using dropsondes and satellitederived atmospheric motion vectors (AMVs; Velden et al. 2005) in a 6-h window around the mission on each analysis day. An isotropic, Gaussian background error covariance was used with the error standard deviations shown in Table 1. The ECMWF analyses were found to have a reasonably good agreement with the observations at the meso- $\alpha$ scale, such that the analyses were not overly sensitive to the prescribed error magnitudes. The Gaussian covariance length scale was set to $4 \Delta x$ in the horizontal to focus on the primary scales resolvable at each nodal resolution. The vertical grid spacing was chosen to be $500 \mathrm{~m}$ for all composites, with a $2 \Delta z$ vertical covariance length scale. A conjugate gradient algorithm was used to minimize an incremental form of the variational cost function with a mass continuity constraint. No other balance or physical constraints were used in the cost function minimization. A background vertical velocity error standard deviation of $1 \mathrm{~m} \mathrm{~s}^{-1}$ constrains the divergence through the mass continuity constraint to be representative of hydrostatic vertical motions.

The 25-km SAMURAI analyses were then used as an initial estimate for the $2-\mathrm{km}$ analyses, and the data window was reduced to $\sim 15 \mathrm{~min}$. The $2-\mathrm{km}$ nodal spacing was chosen to approximately match the alongtrack resolution of the NOAA P-3 tail Doppler radar observations. Dropsondes and satellite-derived wind vectors were included also in the meso- $\beta$-scale analyses to allow finer spatial-scale information to be retrieved from those data, but the wind field was primarily determined from Doppler radar observations. As shown in Table 1, the background error standard deviation was increased to $25 \mathrm{~m} \mathrm{~s}^{-1}$ for all three wind components in the $2-\mathrm{km}$ analyses. The large background error essentially makes the meso- $\beta$-scale analyses independent of the initial estimate where Doppler radar observations were available. Given the poor spatial sampling at this scale from the dropsonde data alone, the analyzed winds were removed in areas with no radar reflectivity.

As a quantitative tool for examining the nature of Karl's spinup, the circulation equation was used to calculate the contributions to changes in circulation during the pregenesis period from the SAMURAI analyses. The form of the equation used in this study was derived by Davis and Galarneau (2009) and is given by 
TABLE 1. Summary of SAMURAI settings and observations for meso- $\alpha$ - and meso- $\beta$-scale analyses.

\begin{tabular}{lcc}
\hline \hline Setting & Meso $\alpha$ & Meso $\beta$ \\
\hline $\begin{array}{l}\text { Horizontal node } \\
\text { spacing }(\mathrm{km})\end{array}$ & 25 & 2 \\
$\begin{array}{c}\text { Horizontal error } \\
\text { covariance } \\
\text { length }(\mathrm{km})\end{array}$ & 100 & 8 \\
$\begin{array}{c}\text { Background } u \text { and } v \\
\text { error } \sigma\left(\mathrm{m} \mathrm{s}^{-1}\right)\end{array}$ & 5 & 25 \\
$\begin{array}{c}\text { Background } w \\
\text { error } \sigma\left(\mathrm{m} \mathrm{s}^{-1}\right)\end{array}$ & 1 & 25 \\
$\begin{array}{c}\text { Background } T \\
\text { error } \sigma\left(\mathrm{K}^{-1}\right)\end{array}$ & 3 & - \\
$\begin{array}{c}\text { Background } q_{v} \\
\text { error } \sigma\left(\mathrm{g} \mathrm{kg}^{-1}\right)\end{array}$ & 1 & - \\
$\begin{array}{c}\text { Observations } \\
\text { analyzed }\end{array}$ & $\begin{array}{c}\text { In situ, dropsondes, } \\
\text { AMVs }\end{array}$ & $\begin{array}{c}\text { In situ, dropsondes, } \\
\text { AMVs, TDR }\end{array}$ \\
\hline
\end{tabular}

$$
\begin{aligned}
\frac{\partial C}{\partial t}= & -\bar{\eta} \tilde{\delta} A-\oint \eta^{\prime} \mathbf{v}^{\prime} \cdot \hat{\mathbf{n}} d l+\oint \omega\left(\hat{\mathbf{k}} \times \frac{\partial \mathbf{V}}{\partial p}\right) \cdot \hat{\mathbf{n}} d l \\
& -\oint(\hat{\mathbf{k}} \times \mathbf{F}) \cdot \hat{\mathbf{n}} d l
\end{aligned}
$$

where $C$ denotes circulation, $t$ denotes time, $\eta$ denotes absolute vorticity, $\delta$ denotes divergence, and $A$ denotes the area of the domain. Overbars represent averaging around the perimeter, primes represent perturbations from the perimeter average, and the tilde represents areal averaging. Additional symbols denote the vertical velocity in pressure coordinates $\omega$, the perturbation $\mathbf{v}^{\prime}$ and total $\mathbf{V}$ horizontal vector winds, friction and subgrid-scale forces $\mathbf{F}$, unit vectors in the normal $\hat{\mathbf{n}}$ and vertical directions $\hat{\mathbf{k}}$, and perimeter length increment $d l$.

This form of the circulation equation results from integrating the flux form of the vertical vorticity equation (Haynes and McIntyre 1987) and decomposing the stretching term into mean and eddy components. As is evident from Eq. (1), the four contributions to the circulation tendency are from the area-averaged horizontal convergence of perimeter-averaged absolute vorticity, eddy fluxes of vorticity at the perimeter, tilting contributions at the perimeter, and Reynolds stress at the perimeter, respectively. As pointed out by Davis and Galarneau (2009), the separation of the first two terms into mean horizontal convergence and eddy fluxes allows for a more straightforward interpretation of contributions from vortex stretching and horizontal advection, respectively. Friction was estimated following the method described in Raymond and López Carillo (2011), using a bulk aerodynamic formula for surface stress that decayed exponentially over a scale height of $1.25 \mathrm{~km}$. Due to the extended length of time between flights, it is not possible to calculate the actual circulation tendency on the left-hand side of Eq. (1) with sufficient accuracy from the observations, but the circulation budget obtained from the summation of the right-hand side allows for insight into the leading-order processes that contribute to vortex spinup.

\section{PREDICT analysis}

\section{a. Time series analysis}

Figure 1a shows the track of pre-Karl from PREDICT research missions (thick blue line) and Karl after it was declared by the National Hurricane Center (NHC) (best track, thin black line). The red stars denote the sweet spot location at $850 \mathrm{hPa}$ deduced from the SAMURAI analyses of each mission's data. The sweet spot is defined by the intersection of the critical latitude (where the zonal flow is equal to the wave phase speed) and the wave trough axis (where the meridional velocity is zero). The SAMURAI analyses were first performed in a comoving frame following the estimated motion of the predepression disturbance from ECMWF analyses. A linear fit of the analyzed positions (thick blue line) was used to derive an average system translation speed for the final SAMURAI analyses (red stars). The analyses shown in the next section are centered on the linear track, but the sweet spot location varies somewhat from mission to mission.

Previous work has typically divided the genesis and further intensification into a series of stages, ranging from a preconditioning phase, genesis phase, and more mature phases of the tropical cyclone life cycle. While these may be useful for delineating the morphological stages in a cyclone's evolution, we note that there is no formal, universally accepted definition for tropical cyclogenesis, but rather a somewhat subjective designation within a continuum of intensifying states that meet certain thresholds of convective organization and wind speed. In this study, we use the operational designation of tropical storm status by NHC to define Karl's genesis time, but a precise definition of genesis will be sidestepped in order to focus on understanding the formation process.

The NHC best track for Karl began on late 13 September, denoted by the "L" in Fig. 1, and was approximately $2^{\circ}$ south of the comoving center. The best track, which uses Earth-relative winds, moved northward over the following day prior to tropical depression (denoted "Lx") and tropical storm designation (denoted by the open hurricane symbol). The genesis location, as marked by the tropical storm designation, is closely located near the pouch sweet spot at 1800 UTC 


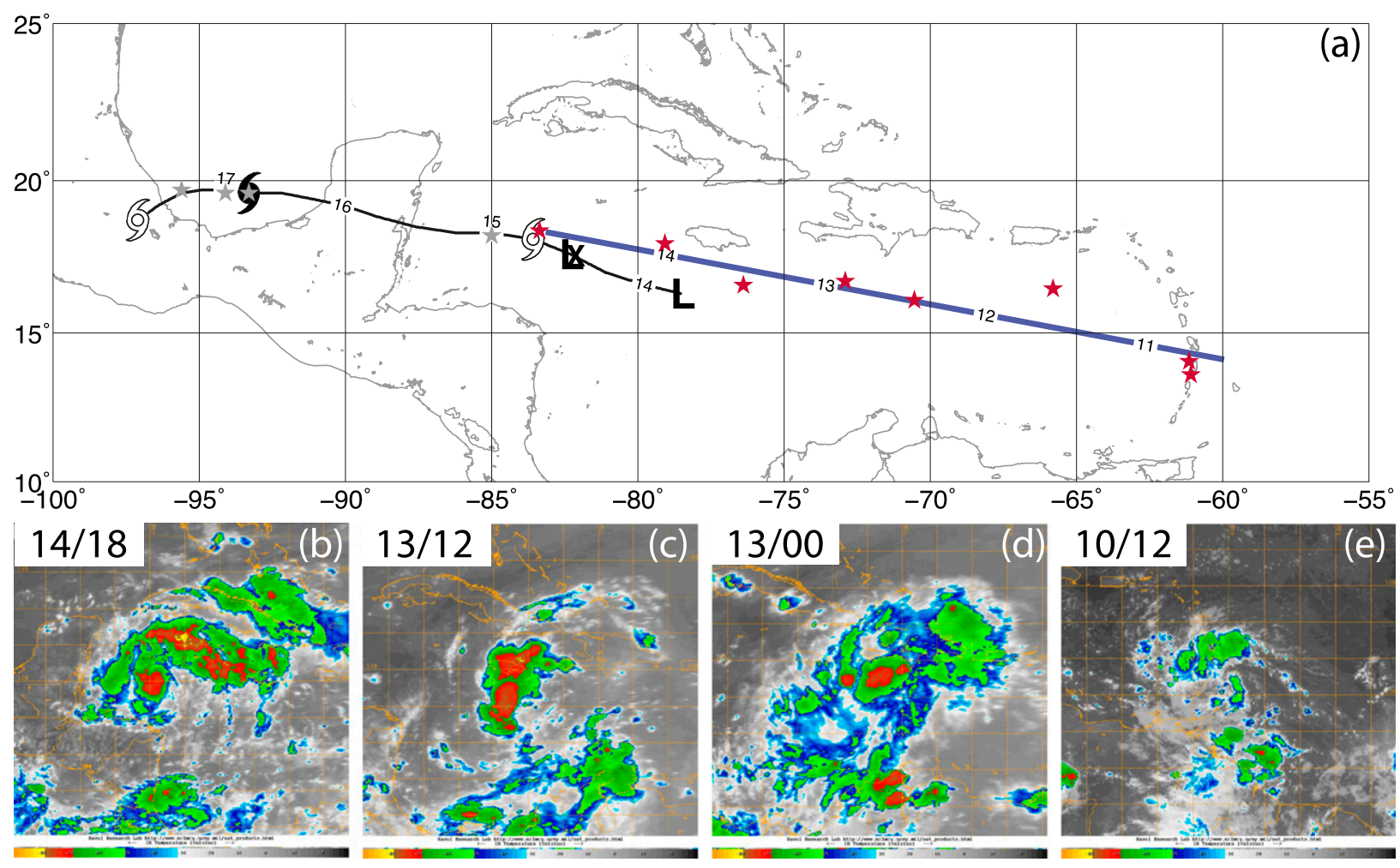

FIG. 1. Track and infrared satellite snapshots of Hurricane Karl. (a) Linear predepression track (thick blue line) derived from 850-hPa pouch centers at mission times (red stars), along with National Hurricane Center best track (thin black line) and storm evolution (L denotes low, Lx denotes depression, open hurricane symbol denotes tropical storm, and closed symbol denotes hurricane). (b)-(e) Infrared satellite imagery at key times. Satellite imagery courtesy of Naval Research Laboratory Monterey.

14 September. Karl continued to move westward as it developed into a hurricane, and was subsequently sampled by IFEX and GRIP research missions (gray stars).

Figures $1 \mathrm{~b}-\mathrm{e}$ show the infrared satellite imagery coincident with some of the aircraft missions leading up to genesis. During the first $\mathrm{GV}$ aircraft mission at 1200 UTC 10 September (Fig. 1e), the cloud cluster was relatively disorganized and composed mostly of cloud tops warmer than $-70^{\circ} \mathrm{C}$ (denoted in green and blue) associated with stratiform MCSs. At 0000 UTC 13 September (Fig. 1d), there were isolated regions of deep convection $\left(<-70^{\circ} \mathrm{C}\right.$ cloud tops denoted in red and yellow) interspersed with warmer cloud tops near the sweet spot. At 1200 UTC later that same day (Fig. 1c), there was a north-south elongated region of active deep convection from $15^{\circ}$ to $19^{\circ} \mathrm{N}$, with a broader region of convective activity well to the south of the sweet spot. This 12-h period is examined in greater detail below. Well-organized deep convection with some banding was evident by 1800 UTC 14 September at the time of genesis (Fig. 1b).

Figure 2 shows a time series of the analyzed meso- $\alpha$ circulation at 850 and $500 \mathrm{hPa}$ over a $600 \mathrm{~km} \times 600 \mathrm{~km}$ domain (Fig. 2a), a $200 \mathrm{~km} \times 200 \mathrm{~km}$ domain (Fig. 2b), and areal fraction of cold-cloud tops from Davis and Ahijevych (2012) (black line). The cold-cloud fraction (CCF) below $-60^{\circ} \mathrm{C}$ was calculated within $200 \mathrm{~km}$ of the model-consensus circulation center, corresponding roughly to the meso- $\alpha$ analysis domain. There was almost no $500-\mathrm{hPa}$ circulation (Fig. 2a, blue triangles) above the $850-\mathrm{hPa}$ circulation (red circles) at 1200 UTC 10 September on the larger meso- $\alpha$ domain. The weak midlevel circulation may be due in part to misalignment of the vortex in the vertical, such that the midlevel circulation was not fully within the analysis domain. A brief intensification of the $850-\mathrm{hPa}$ circulation was seen after the first aircraft mission that was followed by a slow decline over the next several days.

The peaks in CCF represent the time of deep convective bursts, showing the episodic nature of the convection. The aircraft sampled the predepression after a strong convective burst the morning of 11 September when extensive stratiform precipitation was evident in satellite imagery (not shown) and there was a marked increase in 500-hPa circulation analyzed at 1800 UTC 11 September (see Fig. 2). The other periods where the CCF is declining in the time series are generally associated with the convective to stratiform transition. 
9 (a) Mean Circulation over $600 \times 600 \mathrm{~km}$ domain and Cold-cloud Fraction

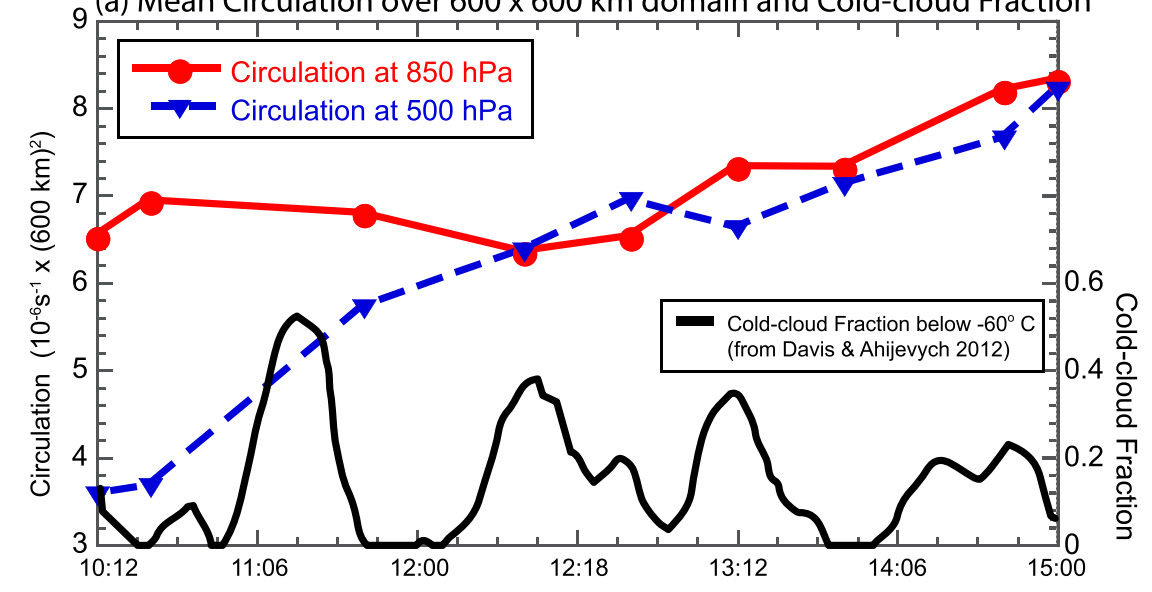

(b) Mean Circulation over $200 \times 200 \mathrm{~km}$ domain and Cold-cloud Fraction

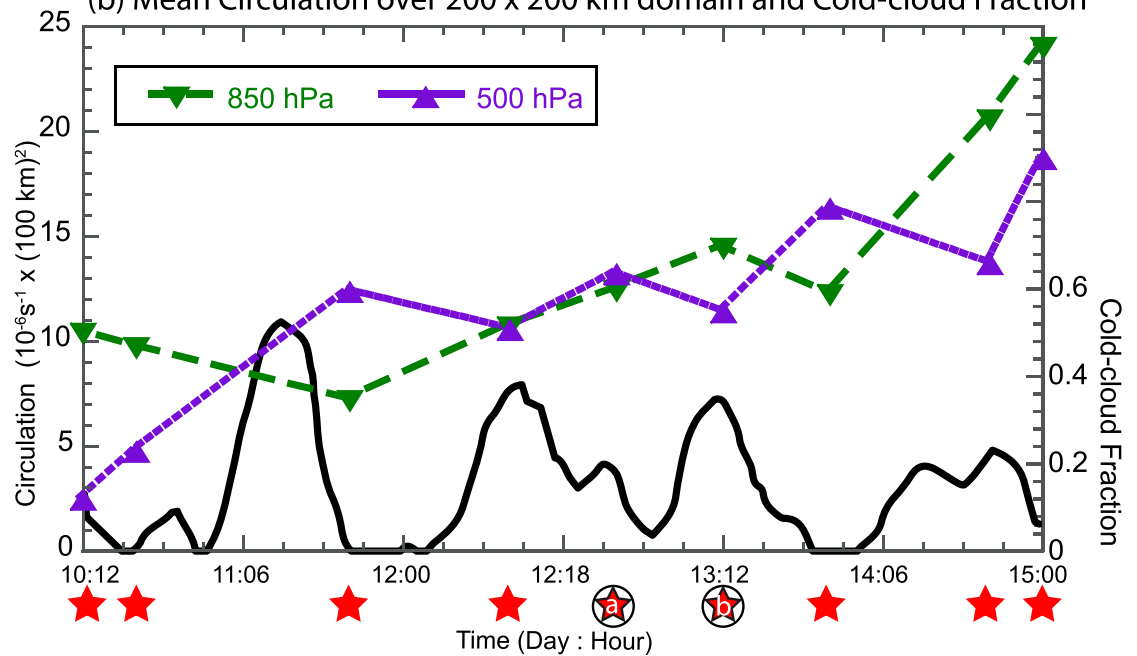

FIG. 2. Analyzed time series of meso- $\alpha$ absolute circulation from the initial aircraft mission to Karl's genesis. Mean absolute circulation at $850 \mathrm{hPa}$ (red solid and green dashed) and $500 \mathrm{hPa}$ (blue long dashed and purple short dashed) over the (a) $600 \mathrm{~km} \times$ $600 \mathrm{~km}$ and (b) $200 \mathrm{~km} \times 200 \mathrm{~km}$ domains centered on the pouch correspond to the left abscissa. The fractional area of cloud tops below $-60^{\circ} \mathrm{C}$ taken from Davis and Ahijevych (2012) corresponds to the right abscissa. Red stars below (b) denote mission times, with 0000 and 1200 UTC 13 Sep highlighted by circled stars.

The linear interpolation of circulation between aircraft missions on 10 and 11 September most likely obscures a more complex time series than depicted. We speculate that the $850-\mathrm{hPa}$ circulation spun down prior to the convective burst, but was followed by some spinup when the deep convection erupted. The net result over this period was a slight weakening of the $850-\mathrm{hPa}$ circulation. A steady, alternating intensification of both the 850 - and $500-\mathrm{hPa}$ circulations followed as the series of convective bursts continued. Genesis declaration by NHC corresponds to the time when the average absolute vorticity in the larger domain exceeded $8 \times 10^{-5} \mathrm{~s}^{-1}$ at both the low and midlevels.
The smaller $200 \mathrm{~km} \times 200 \mathrm{~km}$ domain (Fig. 2b) shows larger amplitude fluctuations in the circulation over time than in the larger domain. Like the larger domain, the initial circulation at $500 \mathrm{hPa}$ was weak but increased significantly as the first convective burst decayed, but the decrease in $850-\mathrm{hPa}$ circulation over the first $18 \mathrm{~h}$ is more evident in the smaller domain. The 850 - and $500-\mathrm{hPa}$ circulations changes are more clearly out of phase on this domain, with the increase at one level occurring at the same time as a decrease at the other level. The circulation increases at $850 \mathrm{hPa}$ tend to occur at the peak of the cold-cloud fraction during the convective bursts, followed by increases at $500 \mathrm{hPa}$ during the stratiform transition period. In the upcoming section 4 a circulation 
(a) Meso- $\alpha$, RH, and winds at $850 \mathrm{hPa}$,

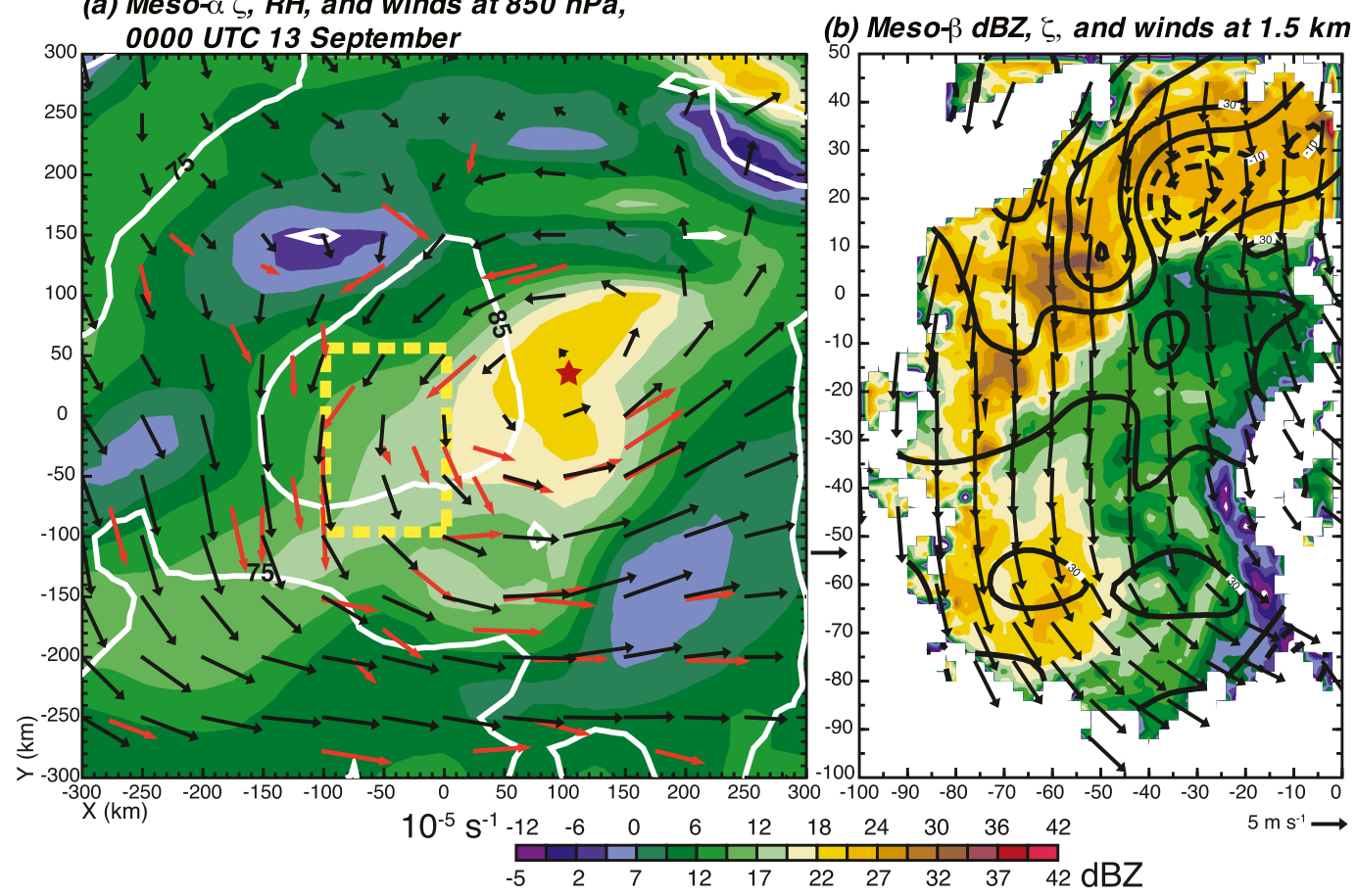

FIG. 3. Low-level meso- $\alpha$ and meso- $\beta$ structure at 0000 UTC 13 Sep. (a) Analyzed meso- $\alpha$ absolute vertical vorticity (color shading, $3 \times 10^{-5} \mathrm{~s}^{-1}$ interval), relative humidity (white, $10 \%$ contour interval), and wave-relative wind vectors (black) at $850 \mathrm{hPa}$. Red vectors indicate measured dropsonde winds. Red star indicates location of the pouch "sweet spot" as defined in the text. (b) Zoomed-in view [yellow box in (a)] of analyzed meso- $\beta$ radar reflectivity (color, $3 \mathrm{dBZ}$ interval), absolute vertical vorticity $\left(20 \times 10^{-5} \mathrm{~s}^{-1}\right.$ contour interval, negative values denoted by dashed lines), and wave-relative wind vectors at $1.5-\mathrm{km}$ altitude. analysis is presented that provides more detail on the circulation changes over the multiple day period leading up to genesis.

\section{b. Meso- $\alpha$ - and meso- $\beta$-scale structure}

The 25-km SAMURAI analyses for all missions have been performed, but here we focus on the analysis at 0000 and 1200 UTC 13 September since (i) this was the only day that quality radar data was available to provide a more detailed look at the meso- $\beta$-scale structure to accompany the meso- $\alpha$-scale analysis, and (ii) we believe the 12 -h evolution on this day is representative of the processes occurring during the predominately convective and stratiform periods at other times. Figure 3 shows the low-level SAMURAI analysis of the meso- $\alpha$ - and meso- $\beta$-scale kinematic, moisture, and reflectivity at 0000 UTC 13 September. For the purposes of this paper, we define "low levels" to be below $700 \mathrm{hPa}$ or 3-km altitude, "midlevels" to be between 700 and $400 \mathrm{hPa}$ or 3-8-km altitude, and "upper levels" to be above $400 \mathrm{hPa}$ or $8 \mathrm{~km}$. To compare with previous studies and simplify the interpretation of the circulation dynamics, the meso- $\alpha$-scale analyses were output on constant pressure levels, with the $850-$ and $500-\mathrm{hPa}$ levels highlighted here. Meso- $\beta$-scale analyses were composed primarily of Doppler radar data that were more appropriate to analyze on constant height levels at approximately the same altitudes of 1.5 and $6 \mathrm{~km}$, respectively.

The analyzed wind in the comoving frame of reference (black vectors) indicates a broad cyclonic recirculating flow on the meso- $\alpha$ scale (25-km nodal mesh) centered around the sweet spot (red star). The red vectors indicate the dropsonde wind observations used in the analysis in the comoving frame. The strongest winds at $850 \mathrm{hPa}$ exceed $\sim 9 \mathrm{~m} \mathrm{~s}^{-1}$ in the comoving frame of reference and are found $\sim 125 \mathrm{~km}$ south-southeast of the sweet spot. The analyzed flow field is in good overall agreement with the observations, with the exception of a few instances. Recall that the analyzed fields incorporate the global model background estimate, observation errors, and mass continuity constraints. For example, $50 \mathrm{~km}$ west and $125 \mathrm{~km}$ north of the origin, denoted here as $(-50,125)$, the dropsonde wind is northwesterly while the analyzed wind is northeasterly. The northwesterly wind would imply strong convergence in the presence of the northeasterly flow indicated by the other nearby dropsondes, and is likely not representative of the meso- $\alpha$-scale flow. 


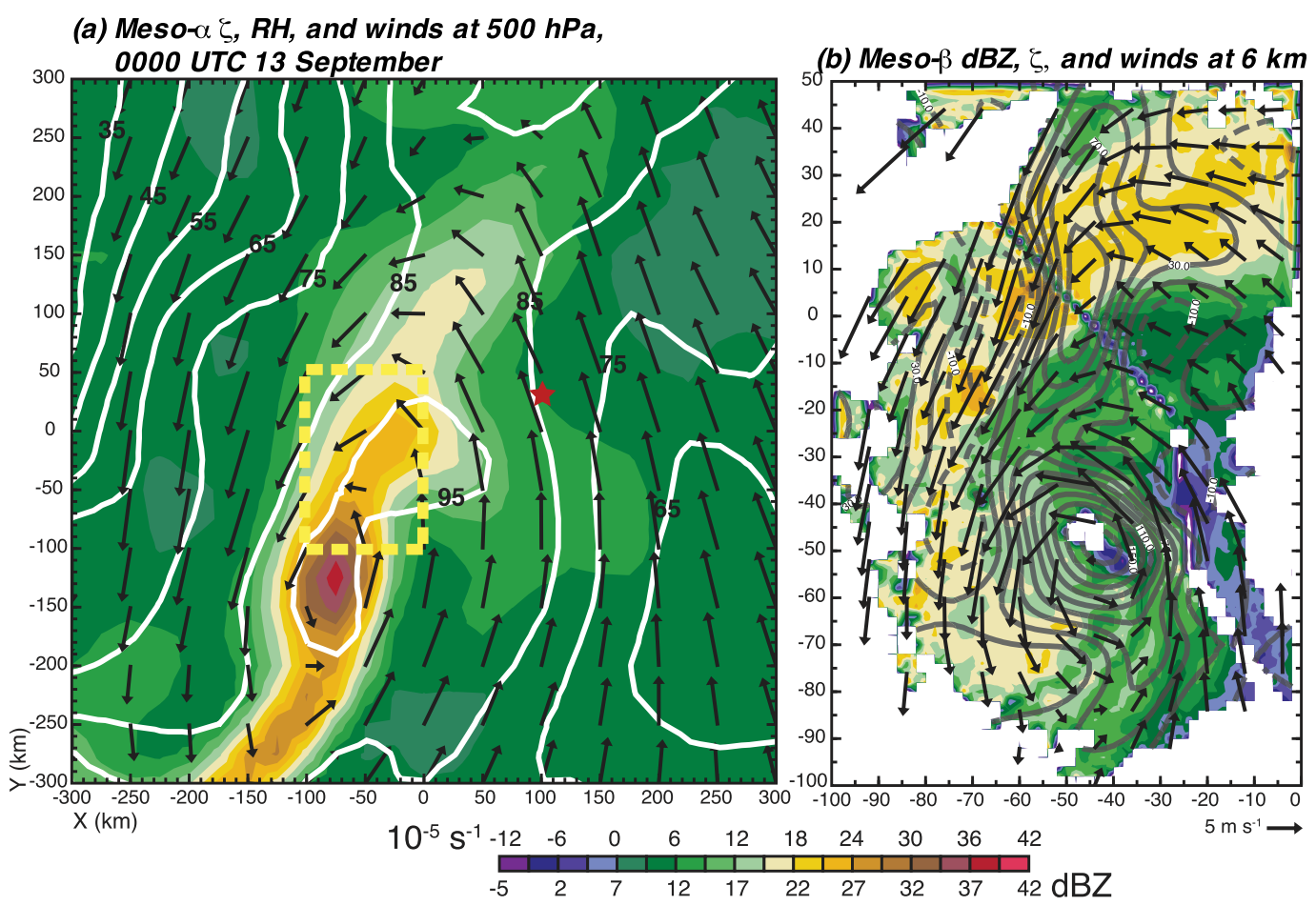

FIG. 4. As in Fig. 3, but for the midlevel meso- $\alpha$ and meso- $\beta$ structure at 0000 UTC 13 Sep and at (a) $500 \mathrm{hPa}$ and (b) $6-\mathrm{km}$ altitude. Absolute vertical vorticity contours in (b) are lightened for clarity.

Figure 3a shows absolute vertical vorticity on the meso- $\alpha$ scale with filled color contours. A southwestnortheast elongated region of positive absolute vorticity had an enhanced central core approximately $200 \mathrm{~km}$ across centered around the sweet spot (red star). Six negative absolute vorticity regions were found on the periphery of the enhanced cyclonic region $125-250 \mathrm{~km}$ away from the sweet spot. The central core of enhanced cyclonic vorticity was greater than approximately 3 times the planetary vorticity at this latitude.

The thick white contours in Fig. 3a indicate $\mathrm{RH}$ contoured in $10 \%$ intervals. A broad region of $\mathrm{RH}$ above $75 \%$ was collocated with the recirculating flow. RH exceeded $85 \%$ in the higher-vorticity region near the sweet spot. The moist pouch center above the boundary layer is consistent with the hypothesis that recirculating air parcels within the disturbance are repeatedly moistened by convective activity as the predepression moved westward.

The dashed yellow box in Fig. 3a indicates the meso$\beta$-scale analysis region in Fig. 3b where Doppler radar observations were collected by the NOAA P3 aircraft. Throughout most of the domain the winds were from the north, but on the southern end of the domain the winds turned cyclonically to the southeast. The winds derived from the Doppler radar are in good agreement with the meso- $\alpha$-scale winds analyzed from the dropsondes and the global analysis. The thick black contours denote isopleths of absolute vorticity. The vorticity in the northern half was composed primarily of shear vorticity, while the vorticity in the southern half was composed primarily of curvature vorticity.

A higher level of detail is apparent in the meso$\beta$ vorticity field in comparison to the meso- $\alpha$ analysis. A southwest-northeast elongated region of vorticity anomalies is evident in the northern half of the analysis domain. There is a strong cyclonic vorticity maximum at $(-55,10)$ with a value exceeding $2 \times 10^{-4} \mathrm{~s}^{-1}$. Adjacent to this cyclonic region are two anticyclonic vorticity regions with maximum values lower than $-1 \times 10^{-4}$ and $-2 \times 10^{-4} \mathrm{~s}^{-1}$, respectively. In the southern half of the analysis domain there is an east-west elongated region of cyclonic vorticity approximately $70 \mathrm{~km}$ in length.

The radar reflectivity is shown in Fig. 3b with filled color contours. A broad region of stratiform precipitation below $35 \mathrm{dBZ}$ covers most of the analysis domain, punctuated by two convective cores near $(-70,-15)$ and $(-60,5)$. The latter convective core was deeper, and appears to be associated with the $-70^{\circ} \mathrm{C}$ cold cloud-top region in the center of Fig. 1d. Satellite animations suggest the convective core was decaying at this time (not shown). The deeper convective core was collocated with one of the strong cyclonic vorticity anomalies. To the northeast of these two cores was a region of 
(a) Meso- $\alpha \zeta$, RH, and winds at $850 \mathrm{hPa}$, 1200 UTC 13 September

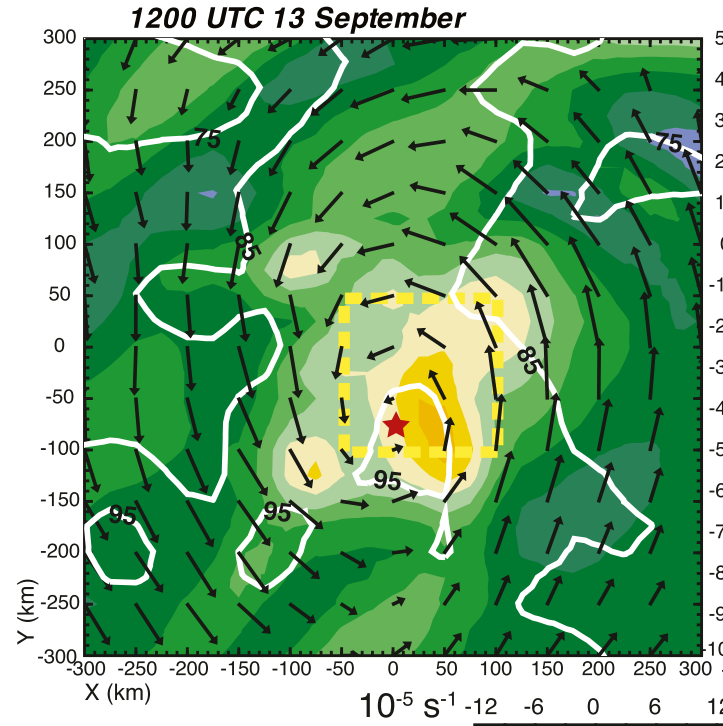

(b) Meso- $\beta d B Z$, $\zeta$, and winds at $1.5 \mathrm{~km}$

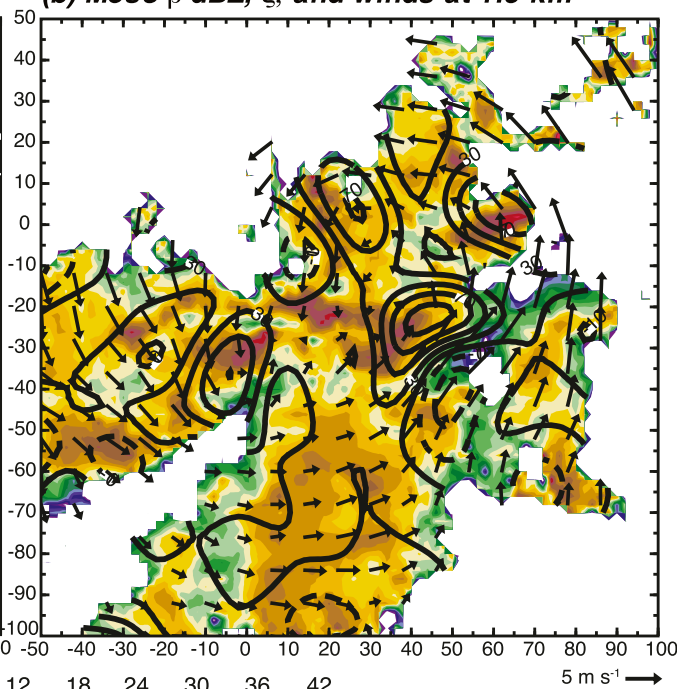

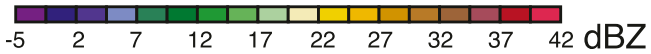

FIG. 5. As in Fig. 3, but at 1200 UTC 13 Sep.

moderate stratiform rain collocated with a stronger anticyclonic vorticity anomaly. Another region of moderate stratiform rain was collocated with the cyclonic curvature in the southern portion of the domain.

Figure 4 shows the $500-\mathrm{hPa}$ and $6-\mathrm{km}$-altitude SAMURAI analyses in similar format to Fig. 3. The recirculating flow is stronger at $6 \mathrm{~km}$ than at $1.5 \mathrm{~km}$, with the local circulation center displaced $\sim 225 \mathrm{~km}$ to the southwest of the $1.5-\mathrm{km}$-altitude sweet spot. The separation of the circulation centers at low and midlevels was likely due to vertical shearing flow of $4 \mathrm{~m} \mathrm{~s}^{-1}$ from 925 to $500 \mathrm{hPa}$ at this time (Davis and Ahijevych 2012; cf. Fig. 10). The recirculating flow was elongated in the north-south direction, and the absolute vertical vorticity field (color) was also elongated largely in the northsouth direction, with two trailing vorticity bands extending north-northeast and south-southwest from a central vorticity maximum that exceeds $3 \times 10^{-4} \mathrm{~s}^{-1}$. The central vorticity core is bordered by regions of negative relative vorticity, but unlike $1.5-\mathrm{km}$ altitude there is no negative absolute vorticity.

The RH field (white contours) was closely aligned with the recirculating flow field and absolute vorticity in the southwest-northeast direction. A region over $600 \mathrm{~km}$ long in the north-south direction where the $\mathrm{RH}$ exceeded $85 \%$ was found near the central vorticity core. The central region that was well sampled by the dropsondes shows peak RH values exceeded $95 \%$. A strong moisture gradient was evident in the east-west direction, with $\mathrm{RH}$ below $65 \%$ to the southeast and below $35 \%$ to the northwest of the midlevel vorticity core. The close coupling of enhanced positive vorticity associated with recirculating flow in the translating reference frame and enhanced moisture within this region provide observational evidence of the protection from dry environmental air within the marsupial pouch at midlevels.

Figure $4 \mathrm{~b}$ shows the Doppler radar analysis for the dashed-box region in Fig. 3a. The broad recirculation region present on the meso- $\alpha$ scale is evident also on the meso- $\beta$ scale. At a more detailed level however, there are some distinct differences. The meso- $\beta$-scale circulation center was located $75 \mathrm{~km}$ north of the derived meso- $\alpha$-scale circulation center. Stronger northerly winds around $15 \mathrm{~m} \mathrm{~s}^{-1}$ are found to the west of the center, with weak westerlies south of the center in the comoving frame.

The vorticity field on the meso- $\beta$ scale depicts a broad region of positive vorticity associated with both curvature and shear contributions, with two distinct maxima near $(-40,-50)$ and $(-40,10)$. The northern maximum exceeded $9 \times 10^{-4} \mathrm{~s}^{-1}$ and was part of an asymmetric vorticity dipole with a corresponding weaker region of negative vorticity to the west that was about $-1 \times 10^{-4} \mathrm{~s}^{-1}$. The southern maximum exceeded $1.5 \times 10^{-3} \mathrm{~s}^{-1}$ and was collocated with the meso- $\beta$ circulation center. The southern maximum was approximately $40 \mathrm{~km}$ across, which is less than two nodes in the meso- $\alpha$-scale analysis. Based on the available data it is unclear whether the meso- $\beta$ center is representative of a distinct circulation or is just a more resolved depiction of the circulation evident on the meso- $\alpha$ scale. 


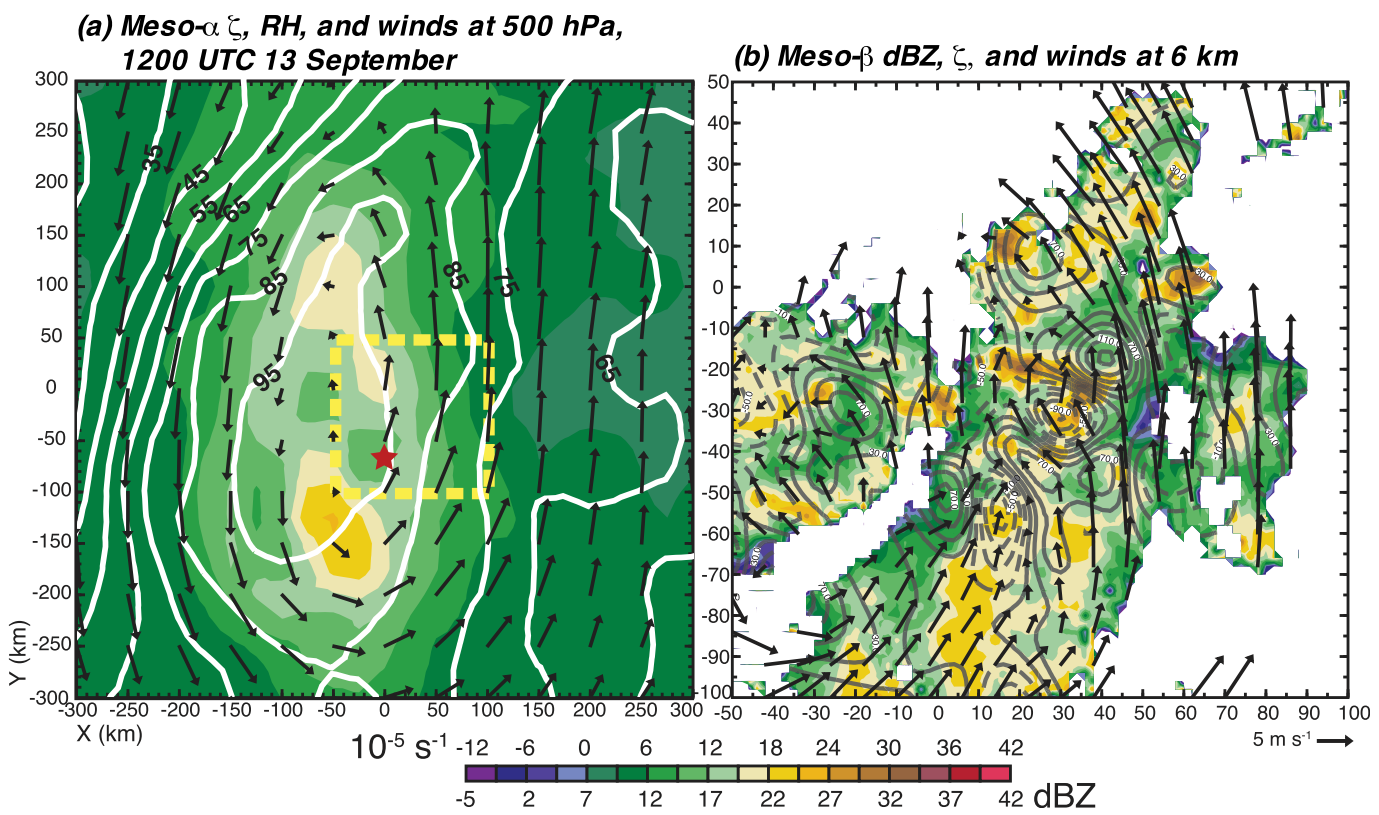

FIG. 6. As in Fig. 4, but at 1200 UTC 13 Sep.

With a stronger suggestion of curved banding in the reflectivity field at $6-\mathrm{km}$ altitude compared to $1.5 \mathrm{~km}$, the reflectivity structure appears to be more congruent with the horizontal winds at $6 \mathrm{~km}$. The decaying convective core discussed in Fig. 3b was approximately centered on the northern asymmetric vorticity dipole. The southern vorticity maximum was collocated with a region of weaker stratiform precipitation.

Twelve hours later, the kinematics, thermodynamics, and precipitation share many similar characteristics with the 0000 UTC structure, but with some important changes relevant to the genesis process. The vorticity increased throughout the meso- $\alpha$-scale domain at $850 \mathrm{hPa}$ (Fig. 5a), with almost no negative absolute vorticity apparent near the sweet spot. RH increased also, with a broader region exceeding $85 \%$ and a smaller region exceeding $95 \%$ that was collocated with the highest vorticity maximum.

Coincident with the increase in vorticity and $\mathrm{RH}$ on the meso- $\alpha$ scale was a change in the vorticity and convective character on the meso- $\beta$ scale (Fig. $5 b$ ). Higher concentrations of positive vorticity were collocated with enhanced reflectivity in convective cores. In contrast to the predominately stratiform precipitation pattern seen at 0000 UTC, the reflectivity had a more cellular appearance and was generally stronger throughout the analysis domain. The significant outbreak of deep convection apparent in the satellite imagery (Fig. 1c) occurred very close to the sweet spot, which provided higher background vorticity for lowlevel vortex stretching in the convective cores. The peak vorticity value at $1.5 \mathrm{~km}$ exceeded $9 \times 10^{-4} \mathrm{~s}^{-1}$ and was nearly twice as high as the observed values at 0000 UTC.

At 6-km altitude however, the peak vorticity on the meso- $\alpha$ scale has decreased by nearly one-half over this 12 -h period (Fig. 6a). The elongated vorticity and $\mathrm{RH}$ region is more compact in the north-south direction, with the $85 \% \mathrm{RH}$ region reduced to $550 \mathrm{~km}$ in length. The strong east-west moisture gradient is still evident. On the meso- $\beta$ scale (Fig. $6 \mathrm{~b}$ ), the cellular convective cores are still apparent at $6-\mathrm{km}$ altitude, and are generally associated with strong vorticity dipoles. Broad recirculating flow is still evident at this scale, but the detailed wind and vorticity fields at $6 \mathrm{~km}$ are more influenced by the convective activity and tilting of horizontal vorticity associated with local vertical wind shear (cf. Bell and Montgomery 2010).

\section{c. Convective and stratiform contributions to genesis}

The SAMURAI analysis at 0000 and 1200 UTC suggests a shift from predominately stratiform to predominately deep convective activity on 13 September that has profound implications for vortex development at these times. The vertical divergence profile of these two precipitation types results in positive and negative vortex stretching at different altitudes that modify the circulation. Vertical cross sections through the meso- $\beta$-scale analysis in Fig. 7 show representative examples of the convection and vorticity that illustrate their relationship. 

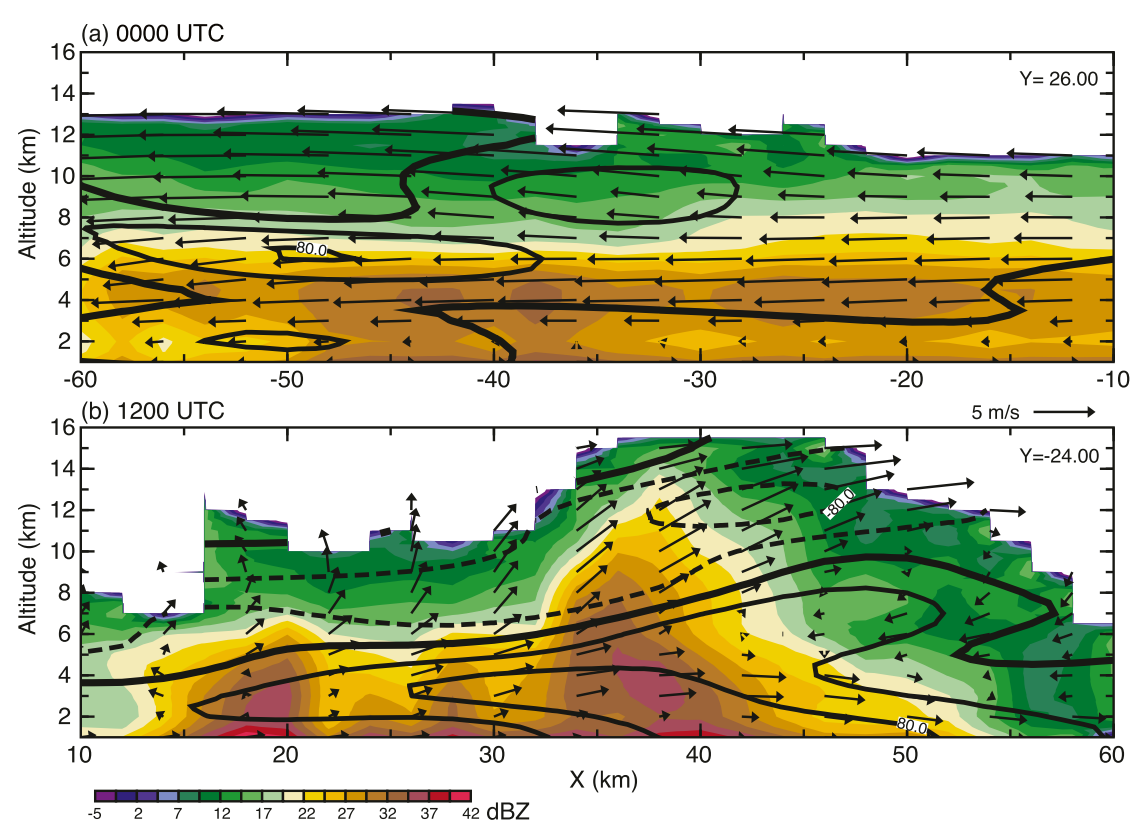

FIG. 7. Radar vertical cross sections showing radar reflectivity (color), absolute vertical vorticity $\left(40 \times 10^{-5} \mathrm{~s}^{-1}\right.$ contour interval, negative values denoted by dashed lines), and wave-relative wind vectors at (a) 0000 and (b) 1200 UTC 13 Sep. Cross sections are taken along $Y=$ (a) 26 and (b) $-24 \mathrm{~km}$ in Figs. 3 and 5, respectively.

The stratiform cross section at 0000 UTC (Fig. 7a) reveals layered echoes, with a broad region of stratiform rain at low levels and weaker echoes aloft associated with ice and snow. The wind vectors show increasing easterly flow with height and only weak vertical velocity, with little indication of individual convective cores. Stratiform precipitation processes produce midlevel convergence (not shown) with weak updrafts above the melting level and downdrafts below it. The cross section indicates a $8 \times 10^{-4} \mathrm{~s}^{-1}$ vorticity maximum near $6 \mathrm{~km}$, suggesting positive stretching of the background vorticity associated with the midlevel convergence.

In contrast, the convective cross section at 1200 UTC (Fig. 7b) reveals cellular echoes, with strong reflectivity near $40 \mathrm{~dB} Z$ at low levels and echo tops reaching $16-\mathrm{km}$ altitude in the deepest convection. Stronger updrafts are apparent in the predominately westerly flow, with some weak easterlies associated with flanking subsidence to the east of the convective tower. Low-level convergence (not shown) associated with the active convection positively stretches local vorticity and results in the maximum vorticity at low levels. Upper-level divergence in the convective outflow compresses vortex tubes and results in a corresponding negative vorticity maximum aloft. Some of the negative vorticity aloft is also likely associated with vorticity dipoles from tilting of horizontal vorticity as seen in Fig. 5b.
The observed convective and vorticity patterns on both the meso- $\alpha$ and meso- $\beta$ scale are consistent with the known divergence profiles and implied vortex stretching for active deep convective and older stratiform precipitation processes (Mapes and Houze 1995). The two precipitation types directly contribute to the low-level and midlevel spinup, respectively. A key question is why the convective character changed over this 12 -h period. To address this question, the difference in the thermodynamic properties of the meso- $\alpha$-scale analyses averaged over the $600 \mathrm{~km} \times 600 \mathrm{~km}$ domain were examined.

Figure 8a shows the vertical profile of average differences of several thermodynamic variables between the two aircraft missions. The differences were calculated by averaging over the meso- $\alpha$-scale domain at 1200 and 0000 UTC and subtracting the mean values. Inflection points near $4-$ and $12-\mathrm{km}$ altitude approximately separate the changes at low, mid-, and upper levels. The potential temperature $\theta$ (green dashed line) decreased slightly at low levels and upper levels and increased slightly at midlevels in the overnight hours. Since the change in the average $\theta$ value represents diabatic effects in clear air and clouds from both radiation and water phase changes, we can only speculate as to the dominant processes. Upper-level cooling is consistent with radiation from cloud tops. The low-level cooling is consistent with both radiation from the surface and 
(a) Thermodynamic Differences

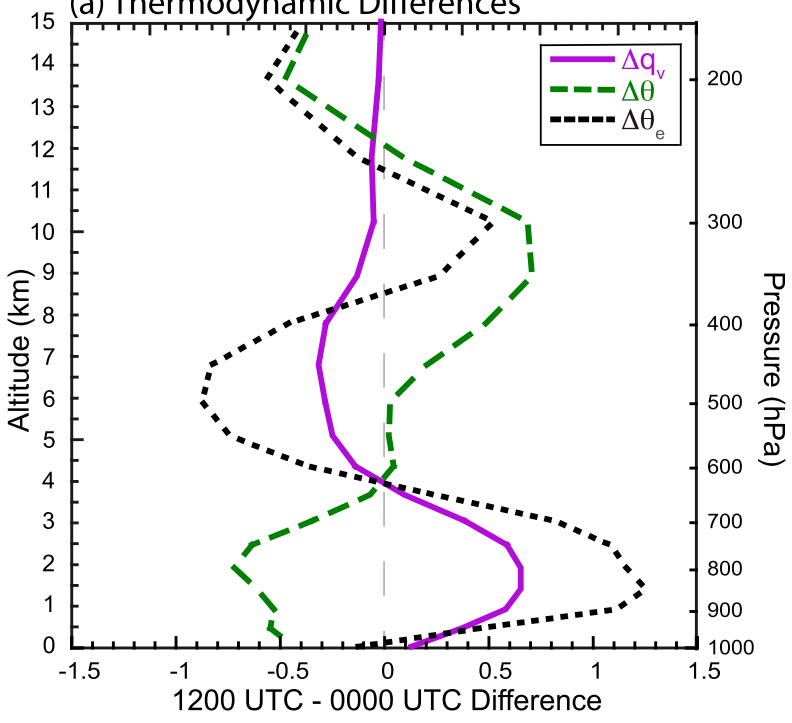

(b) Mean Absolute Vorticity

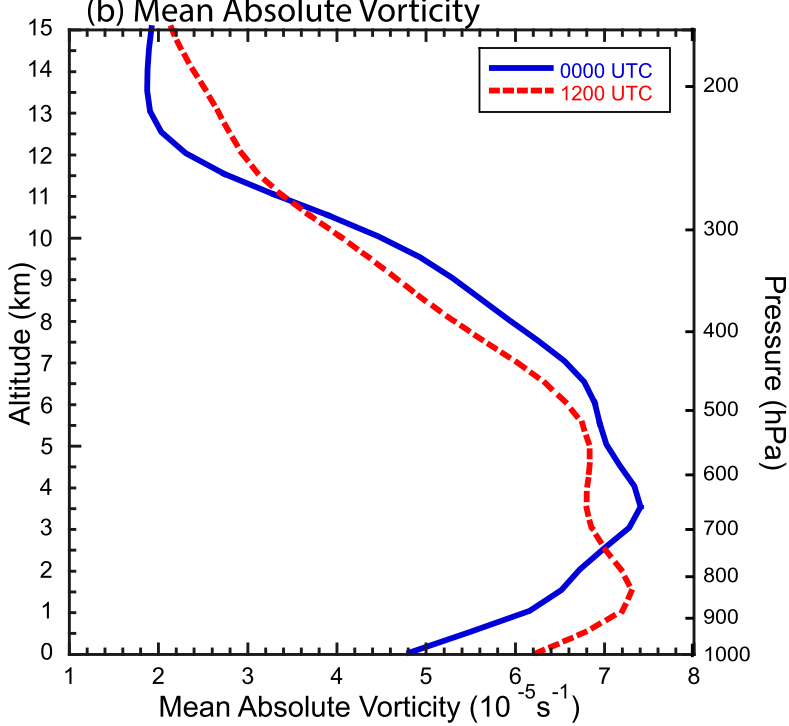

FIG. 8. Thermodynamic and vorticity differences on a $600 \mathrm{~km} \times$ $600 \mathrm{~km}$ domain with height of meso- $\alpha$-averaged quantities from 0000 to 1200 UTC 13 Sep. (a) Difference (1200 UTC minus $0000 \mathrm{UTC}$ ) of vapor mixing ratio (solid purple, $\mathrm{g} \mathrm{kg}^{-1}$ ), potential temperature (dashed green, $\mathrm{K}$ ), and equivalent potential temperature (dotted black, K). (b) Meso- $\alpha$-averaged vorticity at 0000 (solid blue) and 1200 UTC (dashed red).

lower-tropospheric water vapor, as well as evaporative cooling from the widespread stratiform rain. The slight midlevel warming in $\theta$ may be due to longwave warming and latent heating in clouds or balanced temperature responses associated with gradient wind adjustment. As will be shown later, the low-level virtual potential temperature oscillates with a period close to diurnal time scale, while the midlevel temperature generally increases over time.
The water vapor mixing ratio $q_{v}$ (purple solid line) increased at low levels, decreased slightly at midlevels, and showed minimal change at upper levels. These changes are consistent with the microphysical effects of stratiform precipitation. The low-level increase is consistent with rain evaporation in the stratiform MCS, but detrainment from shallow and congestus convection and dynamical impacts from moisture convergence may also have contributed. The decrease in mixing ratio above the freezing level is consistent with vapor deposition of ice crystals, but moisture divergence may also have played a role. Smaller amounts of water vapor at upper levels showed little change. The changes in $\theta$ and $q_{v}$ affect the equivalent potential temperature $\theta_{e}$ (black dotted line) in generally opposite ways. The moisture changes at low to midlevels have a larger effect on $\theta_{e}$ than temperature changes, such that the $\theta_{e}$ increased below 4-km altitude and decreased in the middle troposphere. At upper levels, $\theta$ and $\theta_{e}$ were approximately the same due to limited water vapor capacity.

We argue that the extensive stratiform precipitation in the preceding $12 \mathrm{~h}$ played both a dynamic and a thermodynamic role in aiding the early morning deep convective bursts around 1200 UTC. From a dynamical perspective, stratiform midlevel convergence increased the depth of the recirculating flow and the protective pouch that reduced environmental dry-air intrusions on the circulation. The strong correlation between $\mathrm{RH}$ and vorticity in the meso- $\alpha$-scale analyses supports this hypothesis. From a thermodynamic perspective, evaporative cooling and moistening of the lower troposphere from stratiform rain that was positively phased with diurnal cooling provided an additional benefit for subsequent convection. The dramatic increase in convective activity (Davis and Ahijevych 2012) is consistent with the observed power-law increase in precipitation above a critical value of total column water vapor and saturation fraction (Neelin et al. 2009). Both the onset of deep convection (Holloway and Neelin 2009) and the strength of updrafts and downdrafts (James and Markowski 2010; Kilroy and Smith 2013) have been shown to have a strong relationship with lowertropospheric moisture due to the effects of entrainment on parcel buoyancy above the boundary layer.

The conceptual model presented here bears some similarity with that described by Mapes and Houze (1995) to explain an MCS observed in Tropical Cyclone Oliver (1993). In this model, the deep convective heating results as an opposing response to the thermal anomalies associated with stratiform heating. The stratiform MCS precipitation produces favorable thermodynamic conditions for subsequent deep convection and convective heating that work to eliminate the low-level cool 
(a) 0000 UTC Circulation Tendencies

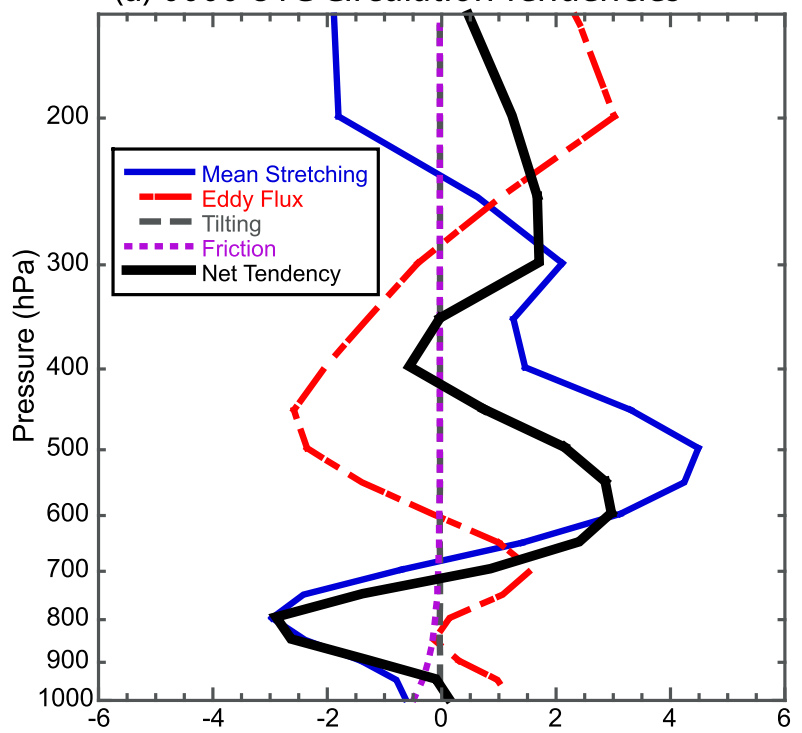

(b) 1200 UTC Circulation Tendencies

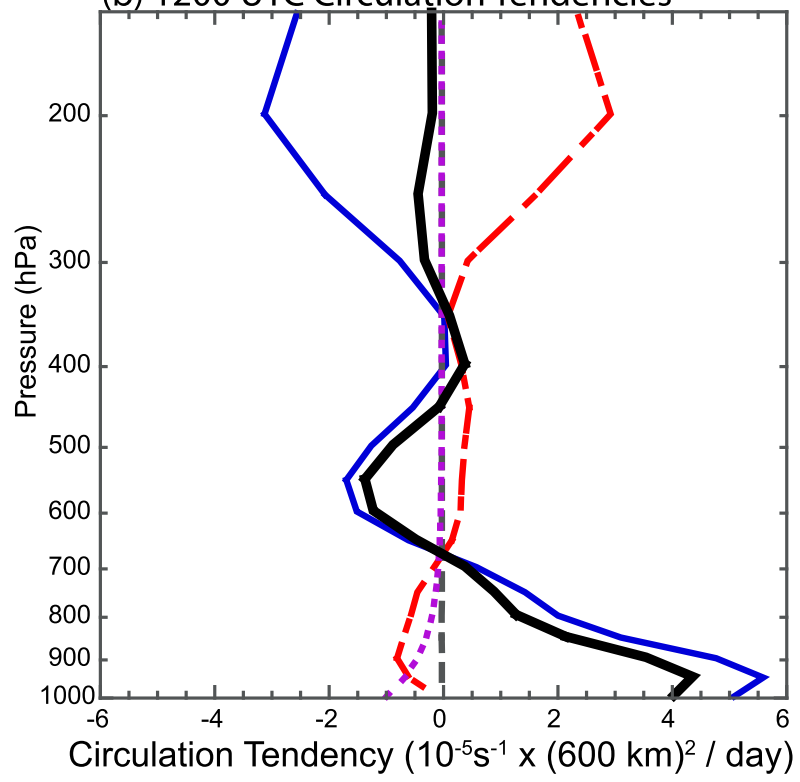

FIG. 9. Meso- $\alpha$-scale $(600 \mathrm{~km} \times 600 \mathrm{~km})$ circulation tendencies with height at (a) 0000 and (b) 1200 UTC 13 Sep. Budget terms are mean stretching tendency (solid blue), eddy flux tendency (dash-dotted red), tilting tendency (long-dashed gray), friction tendency (short-dashed purple), and net tendency calculated by summation of the component tendencies (thick solid black).

anomaly. Favorable phasing with the diurnal cycle appears to have aided these conditions in predepression Karl. A key addition to this conceptual model in the genesis context is the dynamical environment of the pouch, allowing for repeated moistening of air parcels after each convective cycle as the disturbance moves northwestward. As will be shown below, the thermal anomalies oscillated with time in a similar manner to the circulation that is consistent with this conceptual model.

\section{Circulation dynamics on the meso- $\alpha$ scale}

The analyses presented in the previous section focused on a 12-h period on 13 September to illustrate the proposed mechanisms for intensifying the predepression prior to genesis. To provide further evidence that vortex stretching resulting from the convective activity was the primary mechanism for spinup, a circulation budget in the meso- $\alpha$-scale domain was performed.

Figure $8 \mathrm{~b}$ shows the vertical profile of average absolute vorticity over the meso- $\alpha$-scale domain at the two times. The mean vorticity was maximized at $650 \mathrm{hPa}$ at 0000 UTC (blue solid line) and decreased toward the surface. At 1200 UTC (red dashed line) the vorticity increased at low levels and decreased at mid- to upper levels. The changes in the vertical absolute vorticity profile are consistent with the meso- $\beta$ analyses and the vertical divergence profiles of stratiform and deep convective precipitation. The two precipitation regimes alternatively spin up and spin down the mid- and lowlevel circulations, with deep convective bursts that transition to stratiform rain, that then precondition the next convective burst.

The contributions to circulation tendency for these two analyses times are shown in Fig. 9. At both times, the net tendency obtained from summation of the component terms was dominated by the mean stretching term, with nonnegligible contributions from eddy fluxes. Due to the large meso- $\alpha$-scale domain, the average vertical velocity was minimal at the perimeter resulting in no significant contribution from tilting. At 0000 UTC (Fig. 9a), the circulation tendency was dominated by the vertical divergence profile associated with stratiform precipitation. Mean midlevel convergence with low- and upper-level divergence in the cyclonic flow resulted in spinup of the midlevel circulation with spindown above and below. Eddy fluxes generally opposed the stretching tendency, but with a smaller magnitude. Friction contributed to spindown below $800 \mathrm{hPa}$, but was much smaller than the leading-order terms. In contrast to previous interpretations (Bister and Emanuel 1997), the circulation tendency suggests that the large stratiform midlevel circulation was not building down toward the surface, and in fact was negatively impacting the lowlevel circulation.

In addition to the direct spindown of the circulation by friction at the perimeter, frictional forcing induces convergence near the surface and divergence above the boundary layer through an Ekman layer-like flow. The calculated stretching tendency below $800 \mathrm{hPa}$ is likely a 


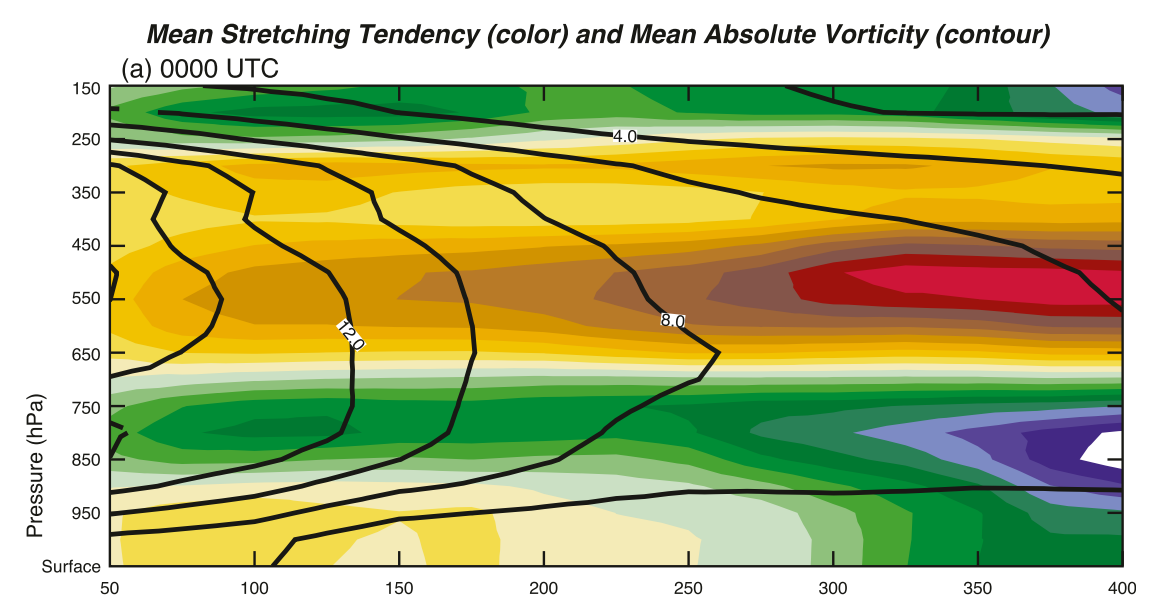

(b) 1200 UTC

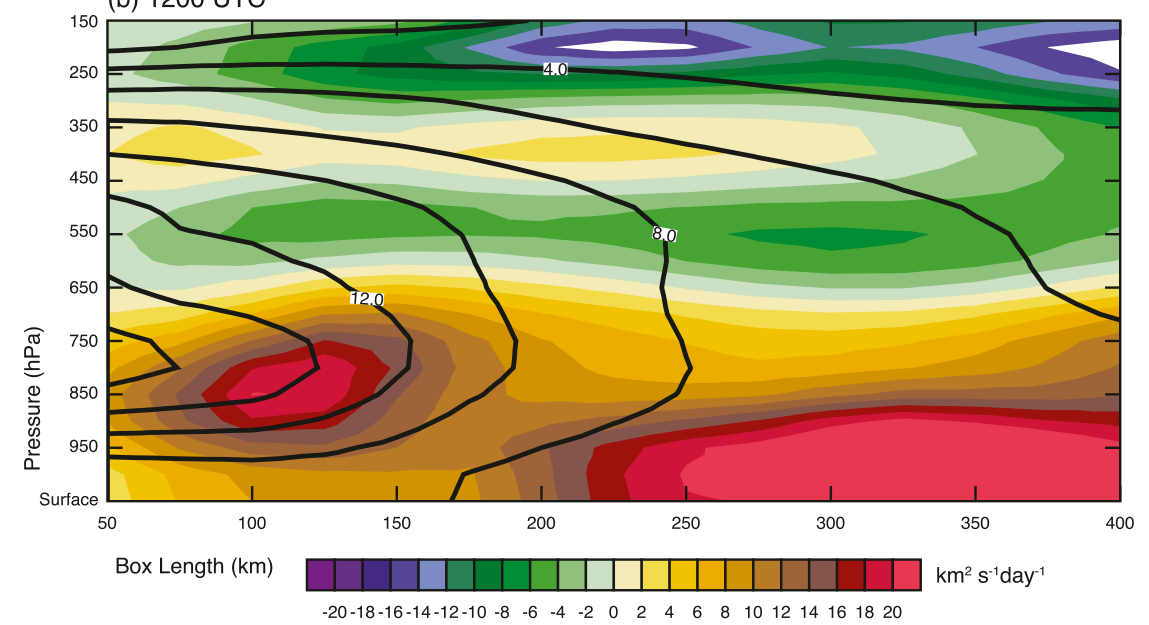

FIG. 10. Mean stretching tendency (color shading, $\mathrm{km}^{2} \mathrm{~s}^{-1}$ day ${ }^{-1}$ ) and absolute vorticity (black contours, $10^{-5} \mathrm{~s}^{-1}$ ) as a function of height and box length at (a) 0000 and (b) 1200 UTC 13 Sep.

combination of both the stratiform divergence and secondary frictional effects. Frictional convergence offsets the stratiform divergence near the surface, but the net effect is a spindown of the vortex above the boundary layer. The circulation tendency suggests that the vortex would lose approximately $35 \%$ of the equivalent circulation of the Coriolis parameter at this latitude in the $12 \mathrm{~h}$ between missions at $850 \mathrm{hPa}$ due to the low-level divergence. However, the circulation tendency was clearly not constant over this period so that is a very rough estimate.

At 1200 UTC (Fig. 9b), the circulation tendency was dominated by the vertical divergence profile associated with convective precipitation. Strong low-level convergence and weak eddy fluxes result in spinup below $700 \mathrm{hPa}$ that is maximized near the surface. Positive tendencies below $700 \mathrm{hPa}$ approximately equal or exceed the spindown seen $12 \mathrm{~h}$ before, consistent with a net low-level spinup over this time period. Mid- and upper-level divergence result in spindown above $700 \mathrm{hPa}$ that is offset by positive eddy fluxes above $300 \mathrm{hPa}$. There are no obvious secondary frictional effects in the profile at this time, but presumably the decreasing convergence with height may be partially a result of these effects. The weaker eddy fluxes may be a result of decreasing vertical shear between 925 and $500 \mathrm{hPa}$ at this time (Davis and Ahijevych 2012).

Lussier et al. (2014) demonstrated that the specific area chosen to calculate the circulation budget can affect the interpretation. To verify that our interpretation is not overly sensitive to the choice of the budget domain, the magnitude of the mean absolute vorticity (e.g., circulation) and mean stretching tendency calculated using different sized boxes is shown in Fig. 10. The sign of the stretching term is largely insensitive to the domain size, except for the region below $950 \mathrm{hPa}$ at 0000 UTC (Fig. 10). The tendency due to mean stretching is slightly above zero if the domain box length is smaller than 

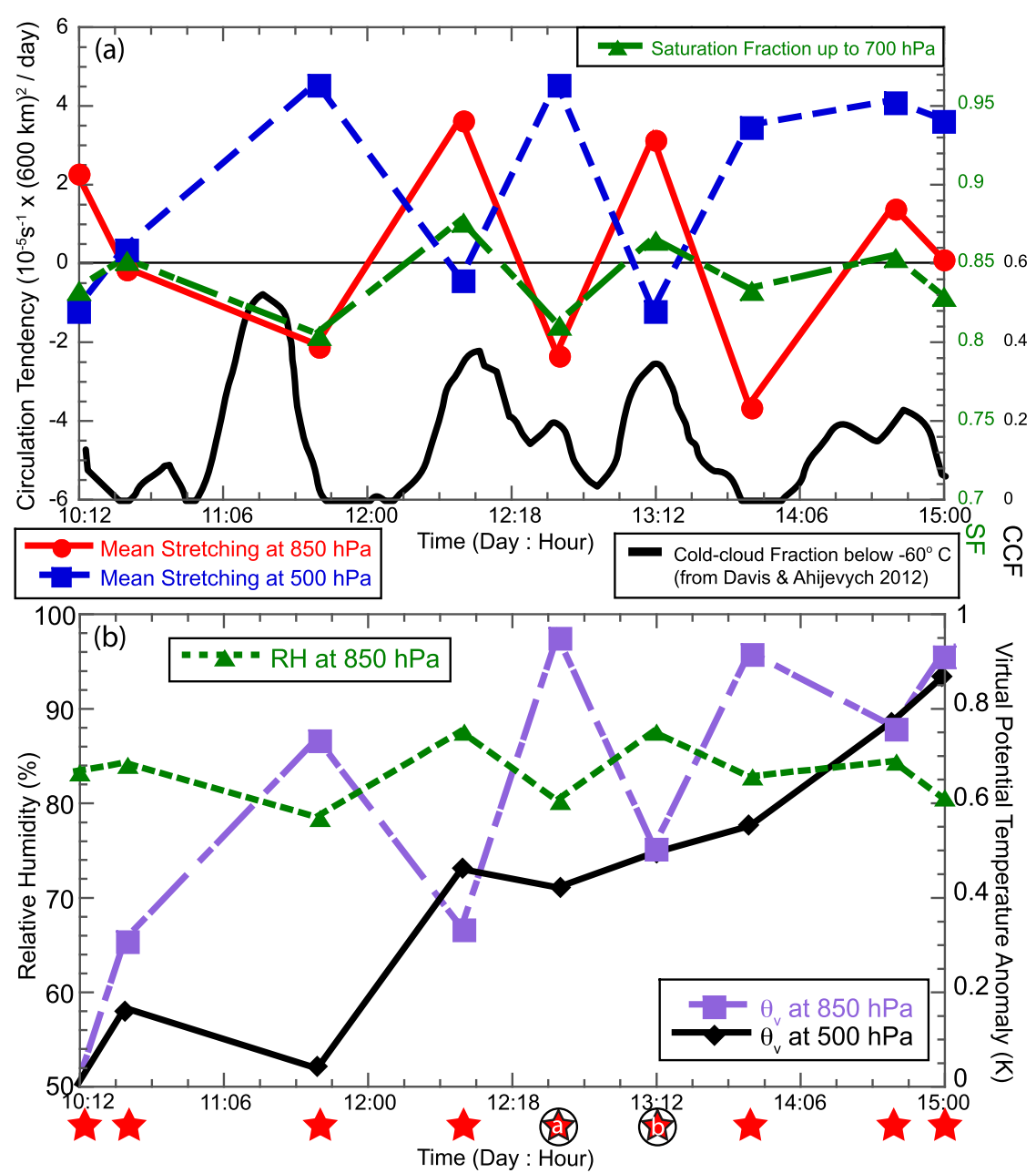

FIG. 11. Analyzed time series of meso- $\alpha$-averaged quantities from initial aircraft mission to Karl's genesis. (a) Circulation tendency $\left[10^{-5} \mathrm{~s}^{-1} \times(600 \mathrm{~km})^{2} \mathrm{day}^{-1}\right]$ at 850 (red circles) and $500 \mathrm{hPa}$ (blue squares) using the left abscissa and saturation fraction up to $700 \mathrm{hPa}$ (green triangles) and cold-cloud fraction below $-60^{\circ} \mathrm{C}$ from Davis and Ahijevych (2012) using the right abscissa. (b) Relative humidity at $850 \mathrm{hPa}$ (green triangles, \%), virtual potential temperature anomaly at $850 \mathrm{hPa}$ (purple squares, $\mathrm{K}$ ), and virtual potential temperature anomaly at $500 \mathrm{hPa}$ (black diamonds, $\mathrm{K}$ ). The anomaly was calculated by removing the mean $\theta_{v}$ from the time series at each respective level. Red stars below (b) denote mission times, with 0000 and 1200 UTC 13 Sep highlighted.

$250 \mathrm{~km}$ from the center (e.g., $500 \mathrm{~km} \times 500 \mathrm{~km}$ across), but is negative using a larger box. However, the general pattern of stronger positive stretching at midlevels and negative or near-zero stretching at low levels does not depend on the domain size.

The structure of the mean absolute vorticity at 0000 UTC is also relatively insensitive to the domain size, but the magnitude does increase as the box gets smaller. The maximum vorticity at midlevels is consistent with the stretching tendency pattern and the presence of widespread stratiform convection. At 1200 UTC, the sign of the stretching term is the same with changing box size except for the region near $400 \mathrm{hPa}$ outside of $300 \mathrm{~km}$. The maximum in positive stretching is slightly higher inside of $150 \mathrm{~km}$, but a deep layer of positive stretching up to $650 \mathrm{hPa}$ is apparent at all domain sizes. The mean vorticity maximum has a similar magnitude at 0000 UTC, and it likewise increases as the box gets smaller. Figure 10 confirms that the circulation tendencies and vorticity structure are not overly sensitive to the domain size, and that the $300-\mathrm{km}$ radius seems to be a reasonable choice for assessing the bulk meso- $\alpha$-scale circulation evolution and tendencies. Using a smaller radius only magnifies the amplitude of the vorticity 
and tendencies, consistent with the evolution depicted in Fig. 2.

The circulation tendencies provide additional evidence that the meso- $\alpha$ circulation was strongly affected by the convective activity, and that a transition from predominately stratiform to deep convective activity occurred over the $12-\mathrm{h}$ period. The analyses support the hypothesis that active deep convection was the primary low-level spinup mechanism and stratiform precipitation was the primary midlevel spinup mechanism, but these illustrations are only snapshots of the predepression period. Observational evidence from PREDICT suggests that more than one convective burst is typically required for genesis to occur (Montgomery et al. 2012). Karl's predepression period was characterized by a series of early morning deep convective bursts followed by stratiform transitions, which are believed to have collectively led to genesis after several days.

A time series of the meso- $\alpha$-scale characteristics leading up to genesis is shown in Fig. 11. The symbols are plotted at the analysis times when research observations were available, denoted by the red stars at the bottom of the figure. The two research missions on 13 September described in detail above are highlighted. In Fig. 9a, the circulation tendencies from mean stretching at 850 (red circles) and $500 \mathrm{hPa}$ (blue squares) show an alternating oscillation through the predepression period. Periods of low-level spinup correspond to periods of midlevel spindown, and midlevel spinup corresponds to low-level spindown. Note that the lines connecting each mission are just linear interpolations, and do not capture higherfrequency changes on the meso- $\alpha$ scale.

The time series of CCF illustrates the relationship between the circulation tendencies and convective activity. The low-level spinup tendencies correspond to the peaks in CCF, strongly suggesting that the low-level stretching was associated with the repeated deep convective bursts. Midlevel spinup tendencies correspond to the time periods shortly after the peak CCF as cloud tops warmed, suggesting that the midlevel stretching was associated with the stratiform transitions.

Saturation fraction (SF) from 1000 to $700 \mathrm{hPa}$ (green triangles) shows the integrated $\mathrm{RH}$ in the lower troposphere. The SF time series indicates a positive, in-phase relationship between available water vapor, the convective bursts, and the low-level spinup tendencies. Active convective periods were characterized by SF above 0.85 , suggesting a possible critical value for the onset of deep convection. The 0.85 value is similar to the critical values reported by Neelin et al. (2009) for tropical convection.

A time series of thermodynamic variables is shown in Fig. 11b. The $\mathrm{RH}$ at $850 \mathrm{hPa}$ (green triangle) oscillated around $85 \%$, similar to the SF integrated through $700 \mathrm{hPa}$ shown in Fig. 11a. The strong relationship between RH at this level and SF is consistent with the results of Holloway and Neelin (2009) that show a maximum in the water vapor variance in the tropics at $850 \mathrm{hPa}$. The virtual potential temperature $\theta_{v}$ anomaly at $850 \mathrm{hPa}$ (purple squares), calculated by removing the mean from the time series, oscillated with the diurnal and convective cycle, but also increased over the predepression period. A diurnal time scale explains $85 \%$ of the variance in $850-\mathrm{hPa} \theta_{v}$ time series, consistent with the conceptual model of diurnal and convective heating and cooling presented earlier.

A weaker $\theta_{v}$ oscillation is seen at $500 \mathrm{hPa}$ (black diamonds) that was out of phase with the low-level temperature oscillation before 13 September, but then increased steadily. The $500-\mathrm{hPa}$ temperature increase is consistent with a balanced temperature response to increasing positive vorticity anomalies at and below this level seen in dropsonde composites of developing disturbances (Davis and Ahijevych 2013; Komaromi 2013; Raymond 2012). The dropsonde composites had cold low-level virtual temperature anomalies compared with various environmental reference states. An important distinction between the SAMURAI analysis of Karl and the composite profiles of those studies is the lack of low-level cooling over the predepression period. The current analyses are more consistent with the results of Smith and Montgomery (2012) that showed warming at all levels over time in their composites. Although the low-level $\theta_{v}$ may indeed be cold compared to nondeveloping disturbances, the temperature trends do not suggest that progressive low-level cooling was important in Karl's genesis process, but rather suggest the importance of episodic low-level cooling that produced favorable conditions for the onset of deep convection.

Comparison of Figs. 11a and $11 \mathrm{~b}$ reveal that increases in $850-\mathrm{hPa}$ circulation are in phase with increases in $\mathrm{RH}$ and $500-\mathrm{hPa} \theta_{v}$, and decreases in $850-\mathrm{hPa} \theta_{v}$. The phasing of the dynamic and thermodynamic variables in the time series is consistent throughout the predepression period. Correlation coefficients between the $850-\mathrm{hPa}$ stretching tendency and $\mathrm{RH}$ time series were above 0.6 at all pressure levels below $700 \mathrm{hPa}$, with a maximum value of 0.78 at the 850-hPa level. Negative correlation with $\theta_{v}$ below $700 \mathrm{hPa}$ with similar magnitudes suggest a prominent role of the diurnal and convective cycle in the meso$\alpha$-scale RH time series. Positive correlations ranging between 0.2 and 0.6 of the stretching tendency with $q_{v}$ time series below $700 \mathrm{hPa}$ (not shown) suggest that moisture recovery was also important to the convective processes. 


\section{Discussion and conclusions}

Observations of the mesoscale processes leading to tropical cyclogenesis were collected as part of the PREDICT, GRIP, and IFEX field campaigns during the summer of 2010. Eight research aircraft missions over the five days prior to the genesis of Hurricane Karl provided Doppler radar, in situ flight level, and dropsonde data documenting the structural changes of the predepression disturbance. The research observations were combined here with satellite atmospheric motion vectors and a priori background estimates of the atmospheric state from the ECMWF $25-\mathrm{km}$ global analyses using a spline-based variational technique called SAMURAI. While the observations were not continuous in time, the SAMURAI analyses at the meso- $\beta$ and meso- $\alpha$ scales following the pre-Karl wave pouch provide a consistent and unprecedented depiction of the evolution of the thermodynamics and dynamics in the predepression period.

Low-level spinup of the meso- $\alpha$-scale cyclonic circulation occurred during periods of more active deep convection, with circulation analysis indicating that the positive tendencies were largely due to vortex tube stretching from mean convergence of perimeter-averaged vorticity. Deep convective periods were also characterized by spindown of the midlevel circulation associated with mean divergence. As the convective bursts waned and transitioned to predominately stratiform mesoscale convective systems, the circulation tendencies transitioned to a complementary signature of low-level spindown and midlevel spinup corresponding with the divergence profile of stratiform precipitation. The changes in meso- $\alpha$-scale circulation due to tendencies from vortex stretching were consistent with the contributions from each predominant precipitation mode. The circulation tendencies for closed circuits that encompass the pouch suggest that the conclusions are not overly sensitive to the choice of the domain size, but the magnitudes of stretching tendencies and circulation increase as the domain size decreases to the meso- $\beta$ scale around the sweet spot region of the pouch, consistent with Wang (2012). Doppler radar analyses on the meso- $\beta$ scale on 13 September confirmed the kinematic and precipitation structure deduced from dropsondes and satellite imagery, and showed the presence of low-level and midlevel vorticity maxima collocated with convective and stratiform precipitation features, respectively.

The low-level and midlevel vorticity and moisture fields appear to be strongly linked throughout the pregenesis period. Specifically, a series of convective bursts occurred near the sweet spot within the highvorticity recirculation region, predominately in the early morning. The meso- $\alpha$-scale thermodynamic environment during the bursts was characterized by enhanced relative humidity in a deep layer, with saturation fraction typically near 0.85 for a layer between the surface and $700 \mathrm{hPa}$. These results are consistent with the concept of a critical threshold for the onset of deep convection in the tropics (Neelin et al. 2009). High relative humidity throughout the lower troposphere would support deep convection by reducing dry-air entrainment, thereby maintaining entraining parcel buoyancy through a deeper layer.

The enhanced relative humidity was due to both reduced potential temperature and enhanced specific humidity. These characteristics are thought to be the result of both diurnal radiational cooling and evaporative cooling in stratiform rain in the overnight hours. The cycle of deep convection and stratiform transitions was in phase with the diurnal cycle, suggesting the time scale for boundary layer moisture and CAPE recovery was similar to the diurnal period consistent with $\mathrm{Li}$ et al. (2006). We speculate that disturbances with forced convective episodes due to stronger vertical shear that are not in phase with the diurnal cycle would be less favorable for development. Further research on the relevant thermodynamic time scales in predepressions and their phasing is recommended.

Composite dropsonde studies from PREDICT have suggested that higher available moisture above the boundary layer was a critical distinguishing factor between developing and nondeveloping disturbances (Smith and Montgomery 2012; Komaromi 2013; Davis and Ahijevych 2013). In some respects, the thermodynamic impact of cooling and moistening by stratiform rain prior to deep convection is similar to that proposed by Bister and Emanuel (1997), but we emphasize here the role of moistening in reducing entrainment in subsequent deep convection (James and Markowski 2010; Kilroy and Smith 2013). Bister and Emanuel (1997, p. 2667) further argue that "the stratiform rain must last long enough to drive the midlevel vortex down to the boundary layer." Our results do not support this hypothesis, and instead suggest that subsequent deep convection is required to spin up the low-level circulation. The low-level spindown associated with the stratiform periods suggest that a negative dynamic impact of the stratiform divergence profile was to weaken the near-surface circulation. We hypothesize that the positive dynamic impact of the stratiform MCSs was to enhance the midlevel pouch through vortex stretching, allowing for protection from the environmental dry air and continued moistening of air parcels through a deeper layer. This indirect effect provided a favorable thermodynamic environment for the repeated onset of deep convection within the parent synoptic-scale wave as it moved northwestward. 
The results from this study suggest the convective cycle in Karl alternately built the low- and midlevel circulations leading to genesis as summarized in the following sequence of events. The initial environment for the development of deep convection was a modest low-level circulation on the meso- $\alpha$ scale with no substantial midlevel circulation on 10 September. A strong convective burst then erupted in the vorticity-rich environment on the morning of 11 September that enhanced the low-level circulation. As the convection waned, the resulting transition to stratiform MCSs strengthened the midlevel pouch through midlevel convergence and vortex stretching associated with ice microphysical processes, and protected the disturbance from the intrusion of dry environmental air. Low-level divergence associated with the stratiform rain acts to spin down the low-level circulation, but surface moisture fluxes and cooling associated with rain evaporation and the diurnal minimum helps to increase the relative humidity, lower the level of free convection, and condition the environment for another burst of deep convection. Once the column saturation reaches a critical value, a subsequent convective burst below the midlevel circulation further enhances the low-level circulation, and the convective cycle repeats.

While cooling from evaporation and radiation appears to have provided an important modulation of temperature, the analysis indicates a warming trend at nearly all levels leading to genesis. The warming was greatest at midlevels, but was nonnegligible at low levels above the boundary layer. We note that the negative temperature anomalies observed by Davis and Ahijevych (2013) and Komaromi (2013) were relative to an environmental reference state, but did not appear to get significantly stronger over time during the predepression period when averaged over all cloud types on a domain with a similar size to that used here. The warming trend in the current analysis is consistent with the analysis of Smith and Montgomery (2012), suggesting that the interpretation of the low-level cold anomaly appears to depend strongly on the analysis technique, domain size, and temporal scale. Our analysis suggests that a balanced increasing warm core in the mid- to upper troposphere was the result of the increasing circulation during the predepression period, and no transition from a cold-core structure was required.

The results suggest further that there was no sustained lowering of the mass flux due to the increased stabilization as hypothesized by Komaromi (2013), Raymond (2012), Gjorgjievska and Raymond (2014), and Davis and Ahijevych (2013). While the analyses are not inconsistent with increasing stabilization as described by the foregoing studies, the results show episodic low-level convergence due to repeated convective bursts within pre-Karl's recirculating pouch region. Our interpretation of the observational analysis presented in this study provides a novel hypothesis for the supporting role of stratiform precipitation in the maintenance of the midlevel cyclonic pouch as a moisture containment vessel within the context of the marsupial paradigm. Our interpretation reaffirms also a primary role of deep convection in the genesis process. The analyses suggest that the onset of deep convection and associated low-level spinup was closely related to the coupling of the vorticity and moisture fields at low and midlevels. Further detailed case studies of developing and nondeveloping storms are necessary to further assess the hypothesis presented herein.

Acknowledgments. This study was supported by the National Science Foundation (NSF) Award AGS0851077. MMB also acknowledges support by the NSF CAREER Award AGS-1701225 and the Office of Naval Research (ONR) Director of Research Early Career Grant N000141613033. MTM acknowledges the support of NSF Grant AGS-1313948, IAA-1656075, NOAA HFIP Grant N0017315WR00048, NASA Grant NNG11PK021, ONR Grant 0001417WX00336, and the U.S. Naval Postgraduate School. The authors thank the PREDICT, GRIP, and IFEX teams for collecting the data used in this study. We would also like to thank David Raymond and two anonymous reviewers for their insightful reviews that improved the manuscript. All code and data used in this study are available upon request.

\section{REFERENCES}

Bell, M. M., and M. T. Montgomery, 2010: Sheared deep vortical convection in pre-depression Hagupit during TCS08. Geophys. Res. Lett., 37, L06802, https://doi.org/10.1029/2009GL042313.

- _ - and K. A. Emanuel, 2012: Air-sea enthalpy and momentum exchange at major hurricane wind speeds. J. Atmos. Sci., 69, 3197-3122, https://doi.org/10.1175/JAS-D-11-0276.1.

Bister, M., and K. A. Emanuel, 1997: The genesis of Hurricane Guillermo: TEXMEX analyses and a modeling study. Mon. Wea. Rev., 125, 2662-2682, https://doi.org/10.1175/15200493(1997) $125<2662$ :TGOHGT $>2.0$. CO; 2 .

Bosart, B. L., W.-C. Lee, and R. M. Wakimoto, 2002: Procedures to improve the accuracy of airborne Doppler radar data. J. Atmos. Oceanic Technol., 19, 322-339, https://doi.org/ 10.1175/1520-0426-19.3.322.

Chang, M., C.-H. Ho, M. S. Park, J. Kim, and M.-H. Ahn, 2017: Multiday evolution of convective bursts during western North Pacific tropical cyclone development and nondevelopment using geostationary satellite measurements. J. Geophys. Res. Atmos., 122, 1635-1649, https://doi.org/10.1002/2016JD025535.

Davis, C. A., and T. J. Galarneau Jr., 2009: The vertical structure of mesoscale convective vortices. J. Atmos. Sci., 66, 686-704, https://doi.org/10.1175/2008JAS2819.1.

, and D. A. Ahijevych, 2012: Mesoscale structural evolution of three tropical weather systems observed during PREDICT. 
J. Atmos. Sci., 69, 1284-1305, https://doi.org/10.1175/JAS-D11-0225.1.

_ and - 2013: Thermodynamic environments of deep convection in Atlantic tropical disturbances. J. Atmos. Sci., 70, 1912-1928, https://doi.org/10.1175/JAS-D-12-0278.1.

Dunkerton, T. J., M. T. Montgomery, and Z. Wang, 2009: Tropical cyclogenesis in a tropical wave critical layer: Easterly waves. Atmos. Chem. Phys., 9, 5587-5646, https://doi.org/10.5194/ acp-9-5587-2009.

Foerster, A. M., and M. M. Bell, 2017: Thermodynamic retrieval in rapidly rotating vortices from multiple-Doppler radar data. J. Atmos. Oceanic Technol., 34, 2353-2374, https://doi.org/ 10.1175/JTECH-D-17-0073.1.

,,-- P. A. Harr, and S. C. Jones, 2014: Observations of the eyewall structure of Typhoon Sinlaku (2008) during the transformation stage of extratropical transition. Mon. Wea. Rev., 142, 3372-3392, https://doi.org/10.1175/MWR-D-13-00313.1.

Frank, N. L., 1970: Atlantic tropical systems of 1969. Mon. Wea. Rev., 98, 307-314, https://doi.org/10.1175/1520-0493(1970) 098<0307:ATSO > 2.3.CO;2.

Freismuth, T. M., B. Rutherford, M. A. Boothe, and M. T. Montgomery, 2016: Why did the storm ex-Gaston (2010) fail to redevelop during the PREDICT experiment? Atmos. Chem. Phys., 16, 8511-8519, https://doi.org/10.5194/acp-16-8511-2016.

Gjorgjievska, S., and D. J. Raymond, 2014: Interaction between dynamics and thermodynamics during tropical cyclogenesis. Atmos. Chem. Phys., 14, 3065-3082, https://doi.org/10.5194/ acp-14-3065-2014.

Gray, W. M., 1968: Global view of the origin of tropical disturbances and storms. Mon. Wea. Rev., 96, 669-700, https://doi.org/ 10.1175/1520-0493(1968)096<0669:GVOTOO >2.0.CO;2.

Haynes, P. H., and M. E. McIntyre, 1987: On the evolution of vorticity and potential vorticity in the presence of diabatic heating and frictional or other forces. J. Atmos. Sci., 44, 828-841, https://doi.org/10.1175/1520-0469(1987)044<0828: OTEOVA $>2.0 . \mathrm{CO} ; 2$.

Hendricks, E. A., M. T. Montgomery, and C. A. Davis, 2004: The role of "vortical" hot towers in the formation of Tropical Cyclone Diana (1984). J. Atmos. Sci., 61, 1209-1232, https:// doi.org/10.1175/1520-0469(2004)061<1209:TROVHT>2.0.CO;2.

Holloway, C. E., and J. D. Neelin, 2009: Moisture vertical structure, column water vapor, and tropical deep convection. J. Atmos. Sci., 66, 1665-1683, https://doi.org/10.1175/2008JAS2806.1.

Houze, R. A., Jr., W.-C. Lee, and M. M. Bell, 2009: Convective contribution to the genesis of Hurricane Ophelia (2005). Mon. Wea. Rev., 137, 2778-2800, https://doi.org/10.1175/ 2009MWR2727.1.

James, R. P., and P. M. Markowski, 2010: A numerical investigation of the effects of dry air aloft on deep convection. Mon. Wea. Rev., 138, 140-161, https://doi.org/10.1175/2009MWR3018.1.

Kilroy, G., and R. K. Smith, 2013: A numerical study of rotating convection during tropical cyclogenesis. Quart. J. Roy. Meteor. Soc., 139, 1255-1269, https://doi.org/10.1002/qj.2022.

Komaromi, W., 2013: An investigation of composite dropsonde profiles for developing and nondeveloping tropical waves during the 2010 PREDICT field campaign. J. Atmos. Sci., 70, 542-558, https://doi.org/10.1175/JAS-D-12-052.1.

Li, T., X. Ge, B. Wang, and Y. Zhu, 2006: Tropical cyclogenesis associated with Rossby wave energy dispersion of a preexisting typhoon. Part II: Numerical simulations. J. Atmos. Sci., 63, 1390-1409, https://doi.org/10.1175/JAS3693.1.

Lussier, L. L., III, M. T. Montgomery, and M. M. Bell, 2014: The genesis of Typhoon Nuri as observed during the Tropical
Cyclone Structure 2008 (TCS-08) field experiment-Part 3: Kinematical and dynamical aspects of genesis. Atmos. Chem. Phys., 14, 8795-8812, https://doi.org/10.5194/acp-148795-2014.

Mapes, B. E., and R. A. Houze Jr., 1995: Diabatic divergence profiles in western Pacific mesoscale convective systems. J. Atmos. Sci., 52, 1807-1828, https://doi.org/10.1175/15200469(1995)052<1807:DDPIWP>2.0.CO;2.

Marks, F. D., Jr., and R. A. Houze Jr., 1984: Airborne Doppler radar observations in Hurricane Debby. Bull. Amer. Meteor. Soc., 65, 569-582, https://doi.org/10.1175/1520-0477(1984) 065<0569:ADROIH > 2.0.CO;2.

McBride, J. L., and R. Zehr, 1981: Observational analysis of tropical cyclone formation. Part II: Comparison of nondeveloping versus developing systems. J. Atmos. Sci., 38, 1132-1151, https://doi.org/10.1175/1520-0469(1981)038<1132: OAOTCF $>2.0 . \mathrm{CO} ; 2$.

Melhauser, C., and F. Zhang, 2014: Diurnal radiation cycle impact on the pregenesis environment of Hurricane Karl (2010). J. Atmos. Sci., 71, 1241-1259, https://doi.org/10.1175/JAS-D-13-0116.1.

Montgomery, M. T., and R. K. Smith, 2012: The genesis of Typhoon Nuri as observed during the Tropical Cyclone Structure 2008 (TCS08) field experiment-Part 2: Observations of the convective environment. Atmos. Chem. Phys., 12, 4001-4009, https://doi.org/10.5194/acp-12-4001-2012.

_ - M. M. Bell, S. D. Aberson, and M. L. Black, 2006: Hurricane Isabel (2003): New insights into the physics of intense storms. Part I: Mean vortex structure and maximum intensity estimates. Bull. Amer. Meteor. Soc., 87, 1335-1347, https://doi.org/ 10.1175/BAMS-87-10-1335.

_ R. K. Smith, and S. V. Nguyen, 2010: Sensitivity of tropical cyclone models to the surface drag coefficient. Quart. J. Roy. Meteor. Soc., 136, 1945-1953, https://doi.org/10.1002/qj.702.

— , and Coauthors, 2012: The Pre-Depression Investigation of Cloud Systems in the Tropics (PREDICT) experiment: Scientific basis, new analysis tools and some first results. Bull. Amer. Meteor. Soc., 93, 153-172, https://doi.org/10.1175/BAMS-D-11-00046.1.

Neelin, J. D., O. Peters, and K. Hales, 2009: The transition to strong convection. J. Atmos. Sci., 66, 2367-2384, https://doi.org/ 10.1175/2009JAS2962.1.

Nguyen, V. S., R. K. Smith, and M. T. Montgomery, 2008: Tropical cyclone intensification and predictability in three dimensions. Quart. J. Roy. Meteor. Soc., 134, 563-582, https://doi.org/ 10.1002/qj.235.

Nolan, D. S., E. D. Rappin, and K. A. Emanuel, 2007: Tropical cyclogenesis sensitivity to environmental parameters in radiative-convective equilibrium. Quart. J. Roy. Meteor. Soc., 133, 2085-2107, https://doi.org/10.1002/qj.170.

Raymond, D. J., 2012: Balanced thermal structure of an intensifying tropical cyclone. Tellus, 64A, 19181, https://doi.org/ 10.3402/tellusa.v64i0.19181.

— tropical cyclogenesis. Geophys. Res. Lett., 34, L06811, https:// doi.org/10.1029/2006GL028607.

— oping Typhoon Nuri (2008). Atmos. Chem. Phys., 11, 147-163, https://doi.org/10.5194/acp-11-147-2011.

_ tropical oceans from environmental conditions. J. Adv. Model. Earth Syst., 8, 703-718, https://doi.org/10.1002/2015MS000595.

— C. López Carillo, and L. L. Cavazos, 1998: Case-studies of developing east Pacific easterly waves. Quart. J. Roy. Meteor. Soc., 124, 2005-2034, https://doi.org/10.1002/qj.49712455011. 
- S. L. Sessions, and C. L. Carrillo, 2011: Thermodynamics of tropical cyclogenesis in the northwest Pacific. J. Geophys. Res., 116, D18101, https://doi.org/10.1029/2011JD015624.

Reasor, P. D., M. T. Montgomery, and L. F. Bosart, 2005: Mesoscale observations of the genesis of Hurricane Dolly (1996). J. Atmos. Sci., 62, 3151-3171, https://doi.org/10.1175/ JAS3540.1.

Sanger, N. T., M. T. Montgomery, R. K. Smith, and M. M. Bell, 2014: An observational study of tropical cyclone spinup in Supertyphoon Jangmi (2008) from 24 to 27 September. Mon. Wea. Rev., 142, 3-28, https://doi.org/10.1175/MWR-D12-00306.1.

Simpson, J., E. Ritchie, G. J. Holland, J. Halverson, and S. Stewart, 1997: Mesoscale interactions in tropical cyclone genesis. Mon. Wea. Rev., 125, 2643-2661, https://doi.org/10.1175/15200493(1997)125<2643:MIITCG > 2.0.CO;2.

Sippel, J. A., J. W. Nielsen-Gammon, and S. E. Allen, 2006: The multiple-vortex nature of tropical cyclogenesis. Mon. Wea. Rev., 134, 1796-1814, https://doi.org/10.1175/MWR3165.1.

Smith, R. K., and M. T. Montgomery, 2012: Observations of the convective environment in developing and non-developing tropical disturbances. Quart. J. Roy. Meteor. Soc., 138, 1721-1739, https://doi.org/10.1002/qj.1910.

Velden, C., and Coauthors, 2005: Recent innovations in deriving tropospheric winds from meteorological satellites. Bull. Amer. Meteor. Soc., 86, 205-224, https://doi.org/10.1175/BAMS-86-2-205.

Wang, Z., 2012: Thermodynamic aspects of tropical cyclone formation. J. Atmos. Sci., 69, 2433-2451, https://doi.org/10.1175/ JAS-D-11-0298.1.
— M. T. Montgomery, and T. J. Dunkerton, 2010a: Genesis of pre-Hurricane Felix (2007). Part I: The role of the easterly wave critical layer. J. Atmos. Sci., 67, 1711-1729, https:// doi.org/10.1175/2009JAS3420.1.

, - - and $\longrightarrow, 2010 \mathrm{~b}$ : Genesis of pre-Hurricane Felix (2007). Part II: Warm core formation, precipitation evolution, and predictability. J. Atmos. Sci., 67, 1730-1744, https://doi.org/ 10.1175/2010JAS3435.1.

- - - and C. Fritz, 2012: A first look at the structure of the wave pouch during the 2009 PREDICT-GRIP dry runs over the Atlantic. Mon. Wea. Rev., 140, 1144-1163, https://doi.org/ 10.1175/MWR-D-10-05063.1.

Wissmeier, U., and R. K. Smith, 2011: Tropical cyclone convection: The effects of ambient vertical vorticity. Quart. J. Roy. Meteor. Soc., 137, 845-857, https://doi.org/10.1002/qj.819.

Zawislak, J., and E. J. Zipser, 2014: Analysis of the thermodynamic properties of developing and nondeveloping tropical disturbances using a comprehensive dropsonde dataset. Mon. Wea. Rev., 142, 1250-1264, https://doi.org/10.1175/MWR-D-13-00253.1.

Zehr, R. M., 1992: Tropical cyclogenesis in the western North Pacific. Ph.D. thesis, Colorado State University, 181 pp.

Zhang, J. A., R. F. Rogers, D. S. Nolan, and F. D. Marks, 2011: On the characteristic height scales of the hurricane boundary layer. Mon. Wea. Rev., 139, 2523-2535, https://doi.org/10.1175/ MWR-D-10-05017.1.

Zipser, E. J., and C. Gautier, 1978: Mesoscale events within a GATE tropical depression. Mon. Wea. Rev., 106, 789-805, https://doi.org/10.1175/1520-0493(1978)106<0789:MEWAGT> 2.0.CO;2. 